\title{
A global map of root biomass across the world's forests
}

3 Yuanyuan Huang ${ }^{1,2^{*}}$, Phillipe Ciais ${ }^{1}$, Maurizio Santoro ${ }^{3}$, David Makowski ${ }^{4,5}$, Jerome Chave ${ }^{6}$,

4 Dmitry Schepaschenko ${ }^{7,8,9}$, Rose Z. Abramoff ${ }^{1}$, Daniel S. Goll ${ }^{10}$, Hui Yang ${ }^{1}$, Ye Chen ${ }^{11}$, Wei

$5 \quad$ Wei $^{12}$, Shilong Piao ${ }^{13,14,15}$

$7 \quad{ }^{1}$ Laboratoire des Sciences du Climat et de l'Environnement, LSCE/IPSL, CEA-CNRS-UVSQ,

8 Université Paris-Saclay, 91191 Gif-sur-Yvette, France.

$9{ }^{2}$ Commonwealth Scientific and Industrial Research Organisation, Aspendale, 3195, Victoria,

10 Australia.

$11{ }^{3}$ Gamma Remote Sensing, 3073 Gümligen, Switzerland.

$12{ }^{4}$ INRA, AgroParisTech, University Paris-Saclay, UMR 211, F-78850 Thiverval-Grignon, France.

$13{ }^{5}$ CIRED, 45bis Avenue de la Belle Gabrielle, 94130 Nogent-sur-Marne, France.

$14{ }^{6}$ Laboratoire Evolution et Diversite Biologique UMR 5174, CNRS, Universite Paul Sabatier, 118

15 route de Narbonne, Toulouse, 31062 France.

$16{ }^{7}$ International Institute for Applied Systems Analysis (IIASA) Schlossplatz 1, A-2361

17 Laxenburg, Austria.

$18{ }^{8}$ Center of Forest Ecology and Productivity of the Russian Academy of Sciences, Moscow

19 117997, Russia

$20{ }^{9}$ Siberian Federal University, Krasnoyarsk, 660041, Russia

$21{ }^{10}$ Department of Geography, University of Augsburg, Germany.

$22{ }^{11}$ Department of Mathematics and Statistics, Northern Arizona University, 86001, Flagstaff, AZ, 23 US.

$24{ }^{12}$ State Key Laboratory of Urban and Regional Ecology, Research Center for Eco-environmental

25 Sciences, Chinese Academy of Sciences, Beijing, 100085, China.

$26{ }^{13}$ Sino-French Institute for Earth System Science, College of Urban and Environmental Sciences,

27 Peking University, Beijing, China

$28{ }^{14}$ Key Laboratory of Alpine Ecology and Biodiversity, Institute of Tibetan Plateau Research,

29 Chinese Academy of Sciences, Beijing, China

$30{ }^{15}$ Center for Excellence in Tibetan Earth Science, Chinese Academy of Sciences,

31 Beijing, China 


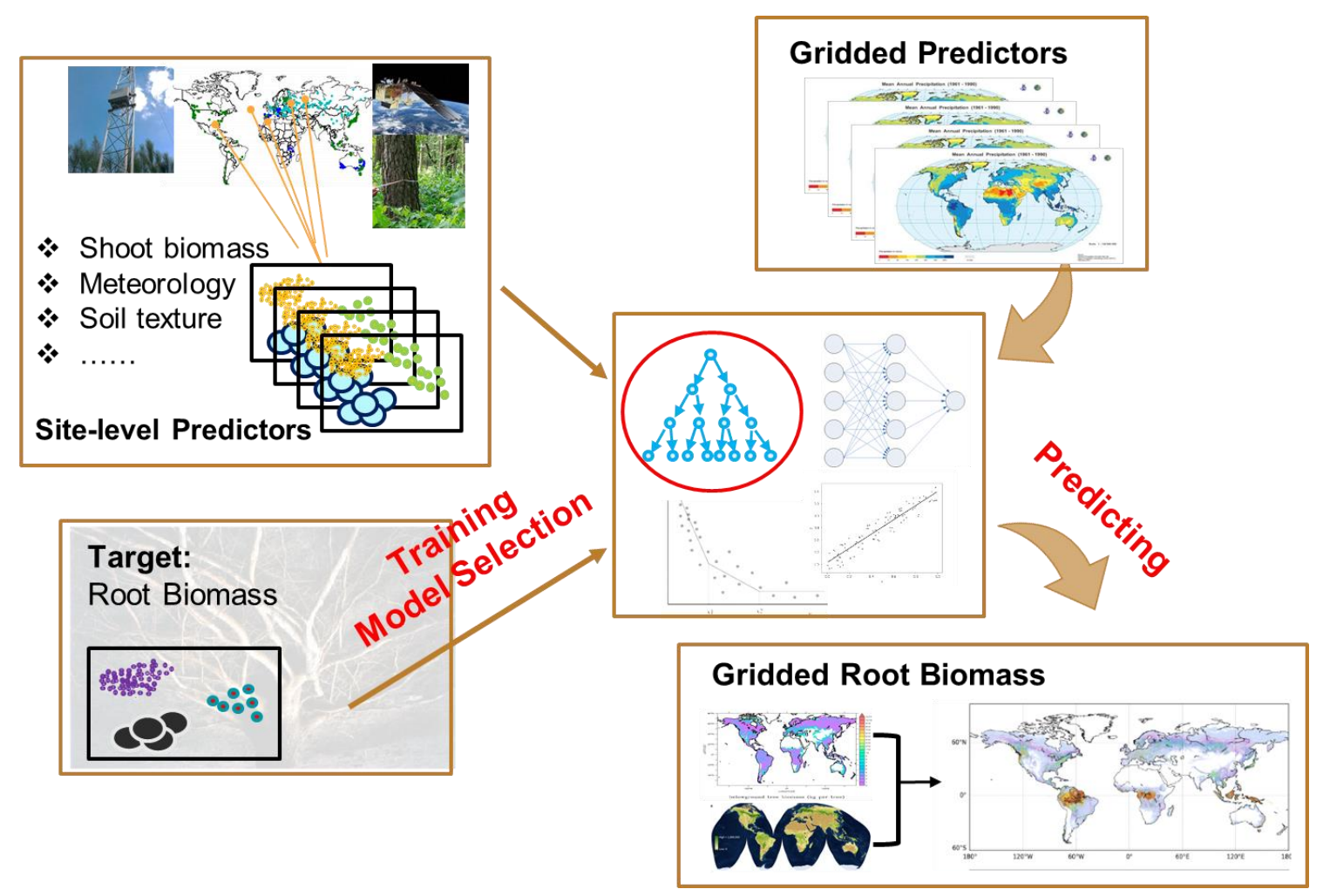

Supplementary Figure 1. Procedures of root biomass mapping at the 1-km resolution. Root biomass mapping is performed in 3 major steps. Step 1: compile field measurements and prepare global gridded predictors; Step 2: train the model with data from Step 1 and select the model with best performance; and Step 3, map root biomass with selected model from Step 2 and gridded predictors from Step 1. We split the data into 3 size categories and selected among 47 predictors through 4 modeling methods (the allometric equation, the random forest, the artificial neural networks and multiple adaptive regression splines). The final root biomass map with a unit of weight per area is created through combining the prediction results (in unit of weight per 


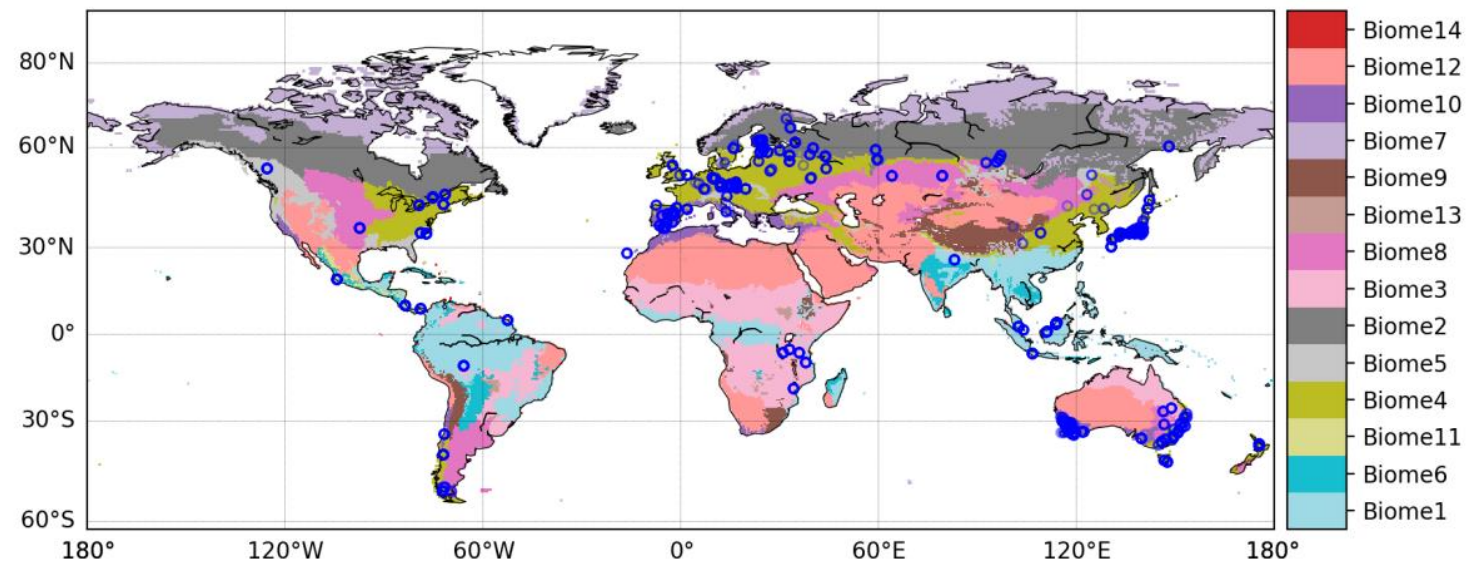

46 Supplementary Figure 2. Geographical distribution of observation sites (blue circles) and biome 47 classes from The Nature Conservancy ${ }^{1}$. Numbers after Biome from the legend are ordered

48 incrementally by decreasing forest area of each biome (Table 3). Biome 1: tropical moist forests;

49 Biome 2:boreal and taiga forests; Biome 3: tropical and subtropical grasslands, savannas and

50 shrublands; Biome 4: temperate broadleaf and mixed forests; Biome 5: temperate coniferous

51 forests; Biome 6: tropical dry forests; Biome 7: tundra; Biome 8: temperate grasslands, savannas

52 and shrublands; Biome 9: montane grasslands and shrublands; Biome 10: Mediterranean forests,

53 woodlands and scrubs; Biome 11: tropical and subtropical coniferous forests; Biome 12: deserts

54 and xeric shrubland; Biome 13: flooded grasslands, savannas; and Biome 14: mangroves. 

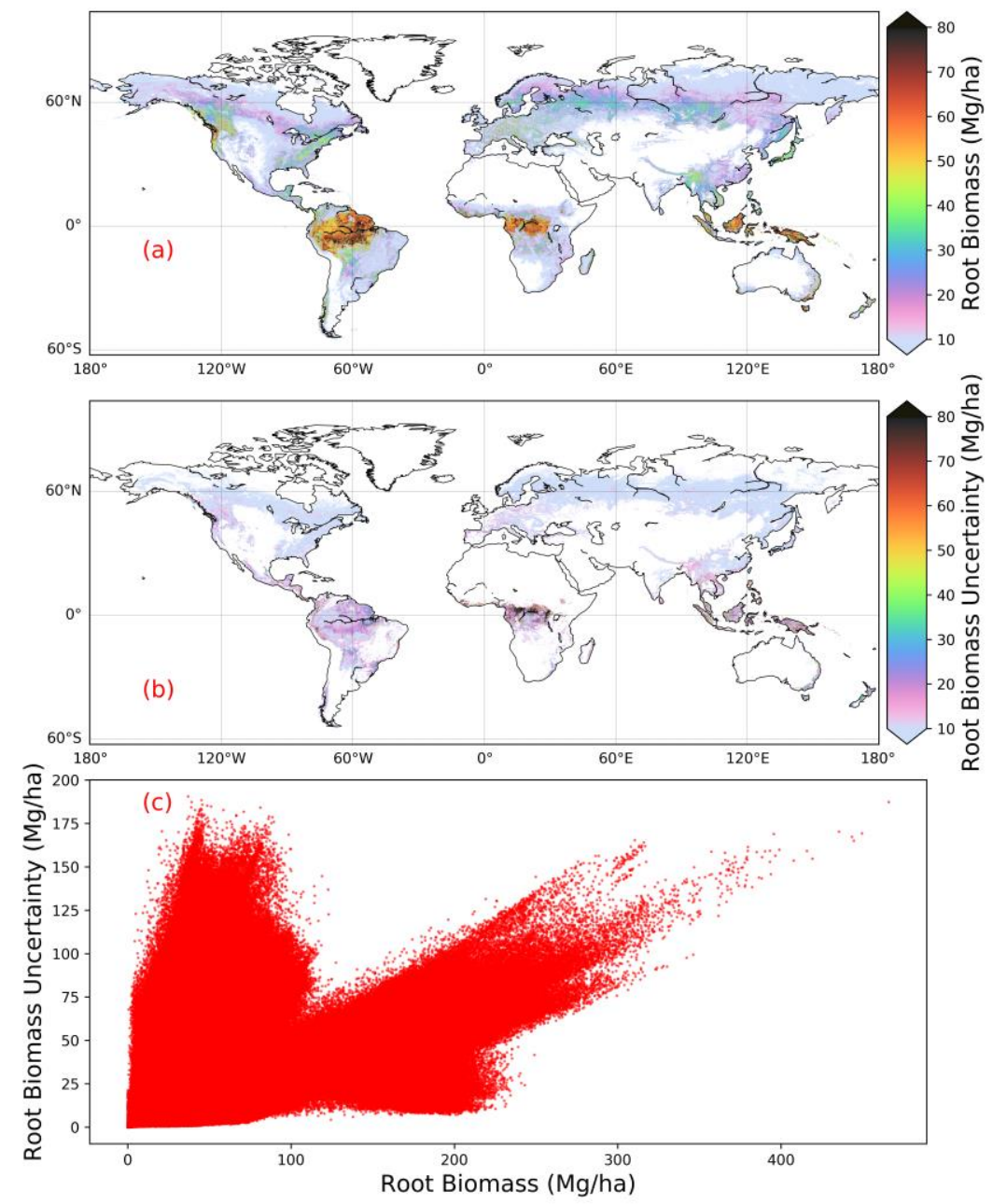

58

59 Supplementary Figure 3. Spatial distribution of (a) root biomass and (b) mapping uncertainty 60 (standard deviation) at $1 \mathrm{~km}$ spatial resolution, and (c) the scatter plot of root biomass vs.

61 mapping uncertainty.

62

63 
(a) Predicting

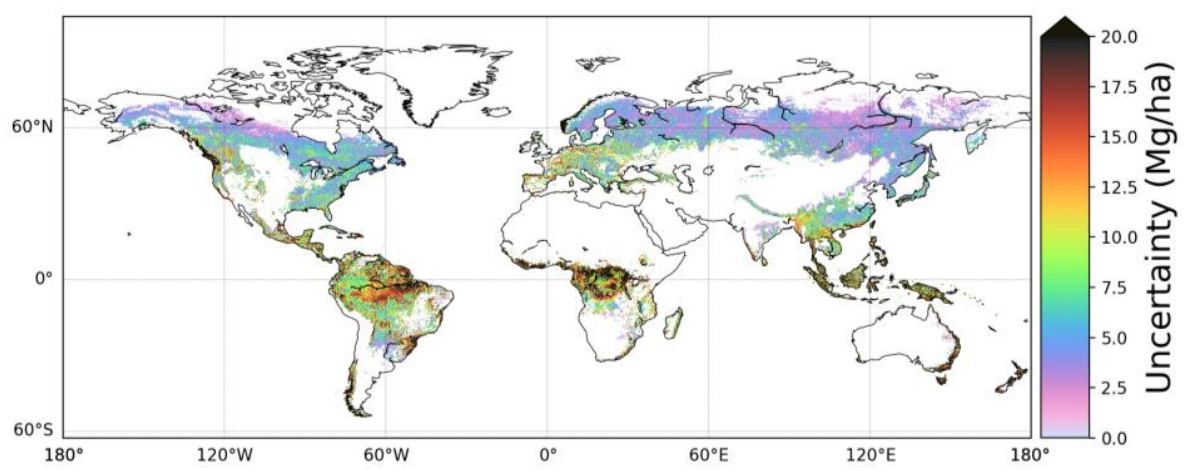

(b) Converting

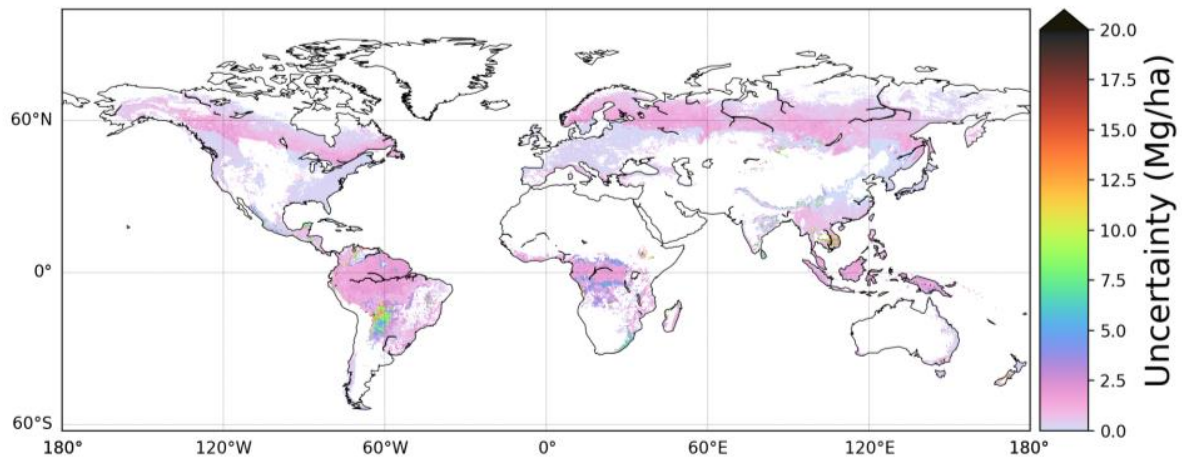

64

65 Supplementary Figure 4. Standard deviations in root biomass mapping due to (a) random forest 66 prediction (a) and (b) unit converting.

67

68

69 

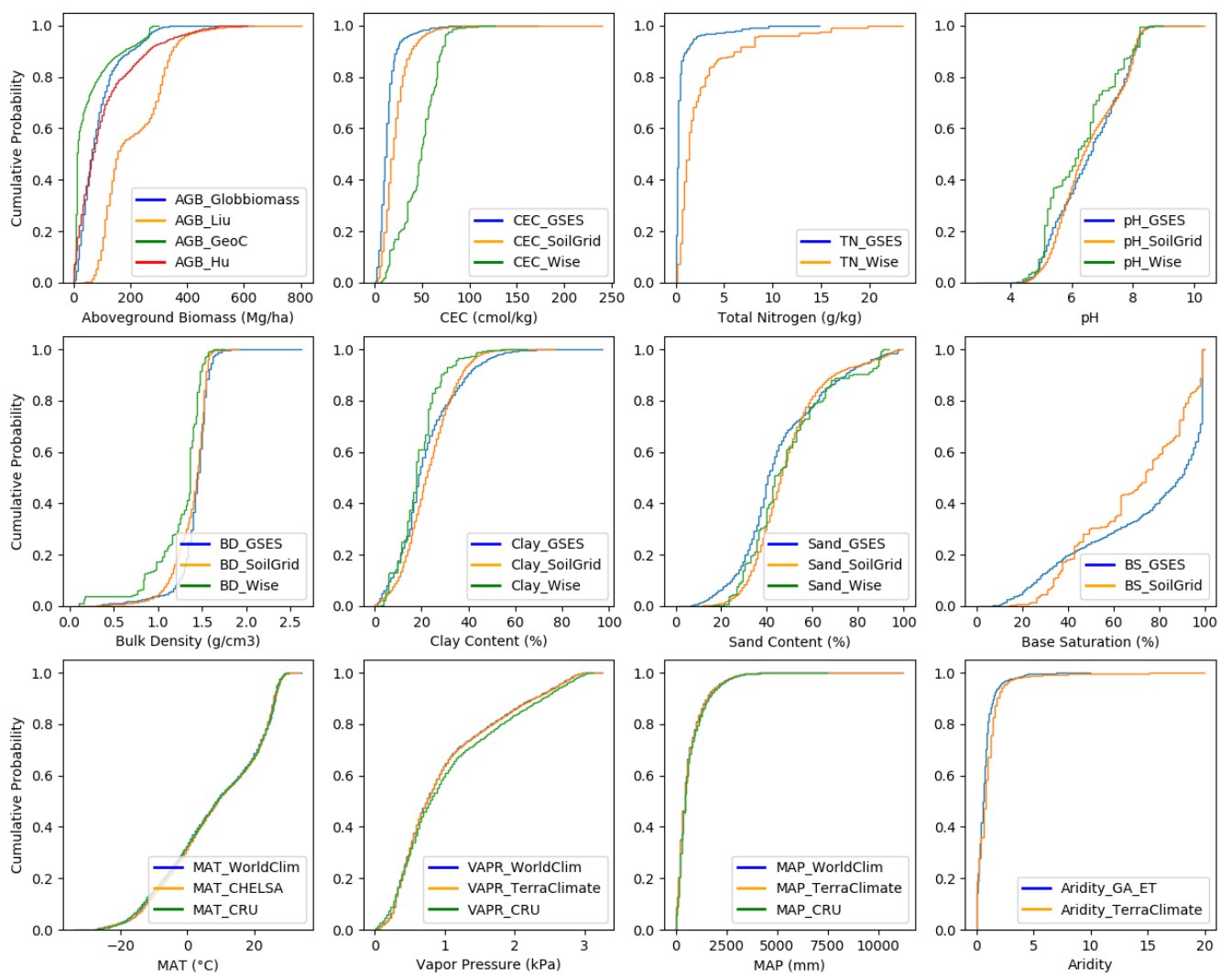

71 Supplementary Figure 5. Cumulative distributions of predictors. Each panel corresponds to one 72 predictor used in quantifying the contribution of random forest prediction uncertainty in root 73 biomass mapping (Supplementary Figure 4a). Different colors indicate different sources for each 74 predictor. Detailed information of data sources is provided in Tables 1, 2. 

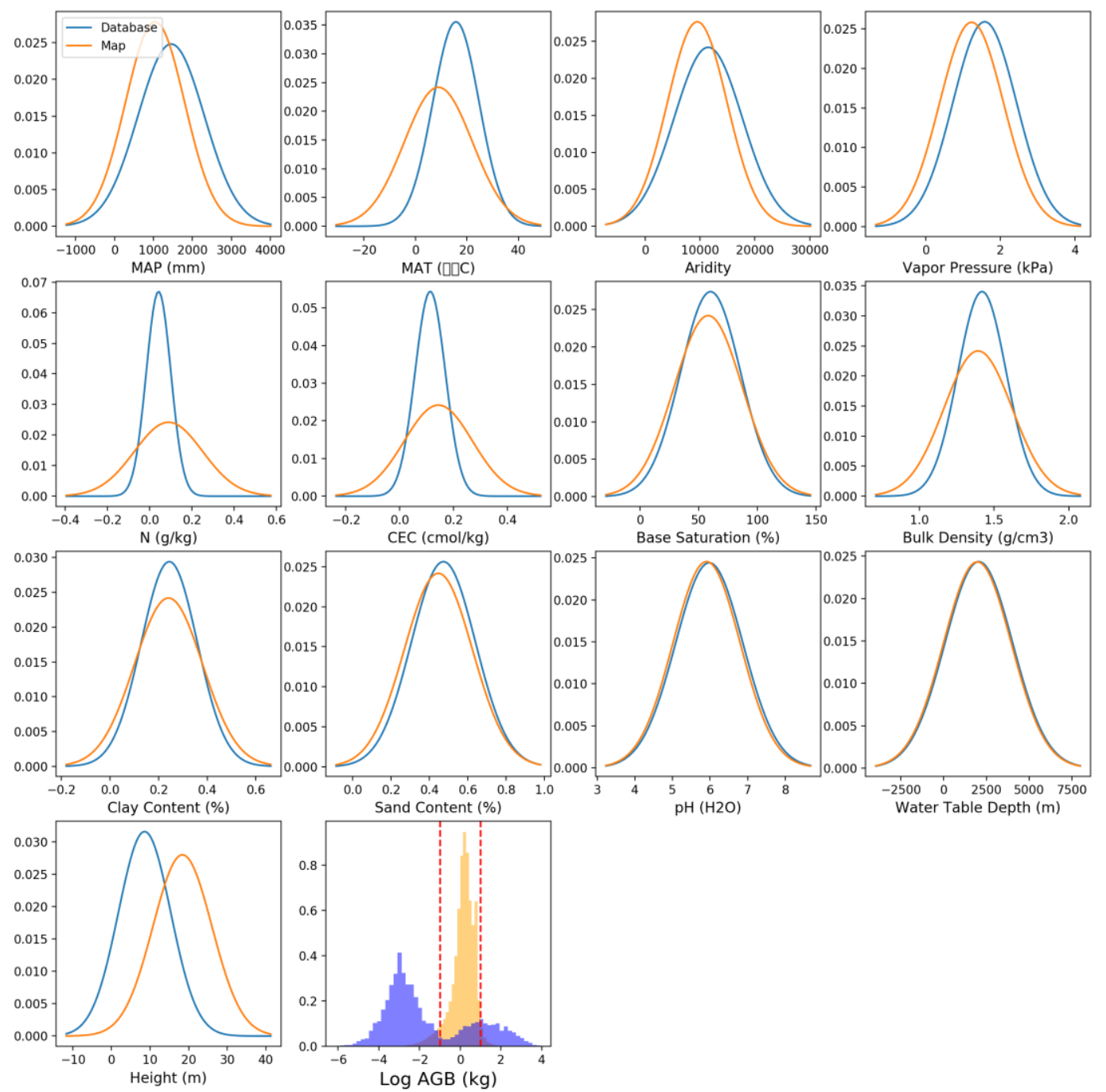

80 Supplementary Figure 6. Distributions of the predictors in the training dataset (blue) and in the 81 global dataset (orange) used to derive the global map. Red dotted lines indicate breakpoints 82 where we separated the datasets for random forest model training and prediction.

83

84 

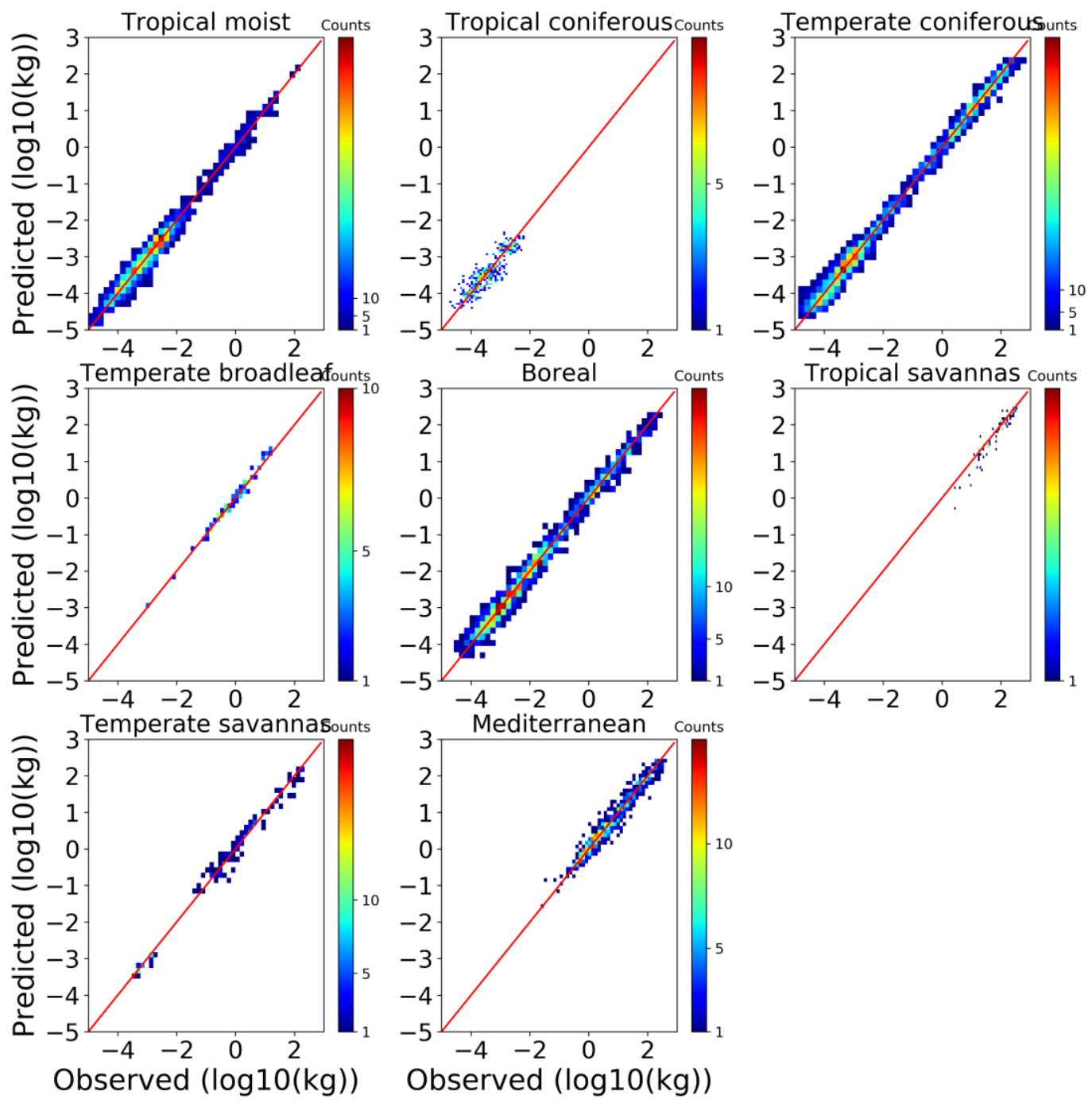

Supplementary Figure 7. Heat plots of predicted root biomass vs. observation at the biome level.

87 Biome classification is from The Nature Conservancy ${ }^{1}$ and is shown in Supplementary Figure 2.

88 The red line is the $1: 1$ line. 

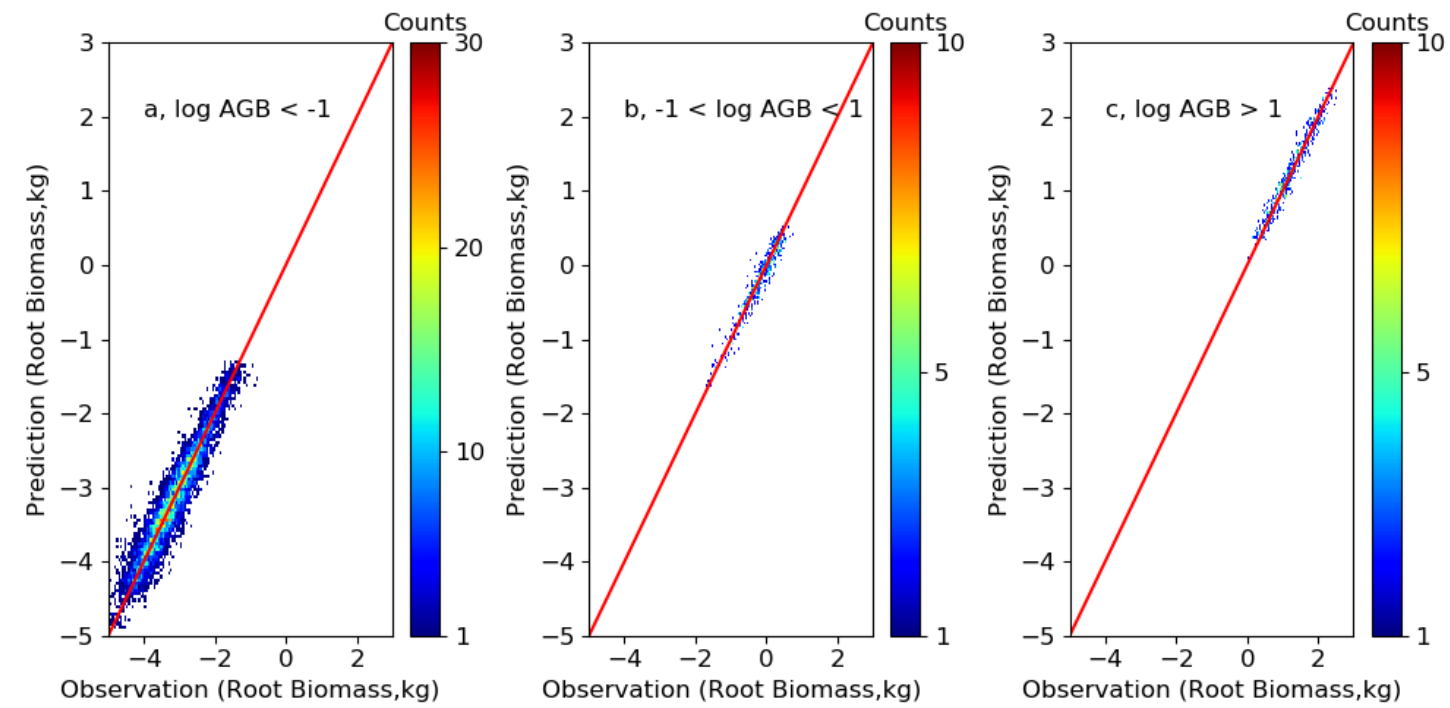

90

Supplementary Figure 8. Heat plots of predicted root biomass vs. observation at different tree

92 sizes. Values are plotted at the log-scale (base 10). The red line is the 1:1 line.

93
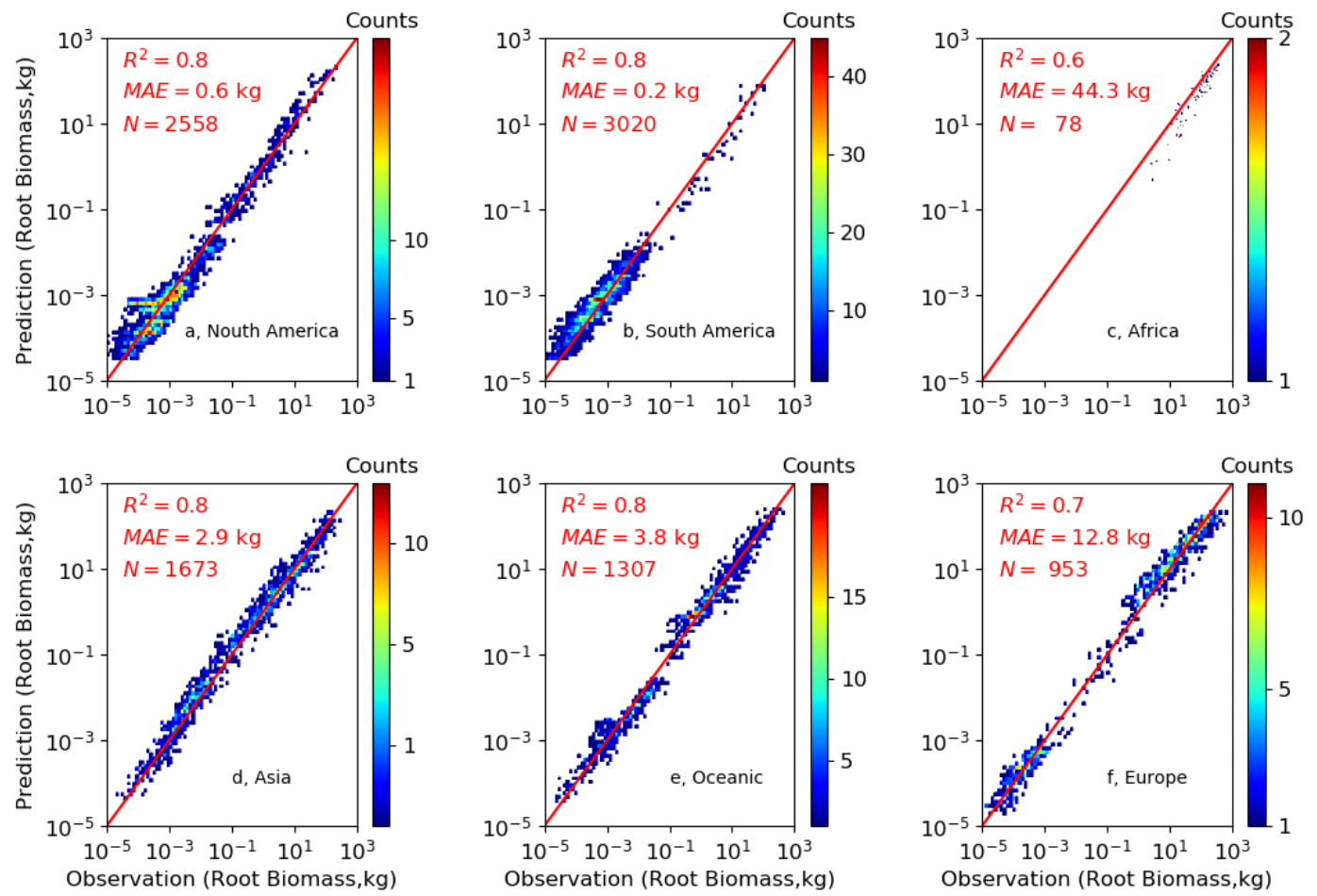

94

95 Supplementary Figure 9. Heat plots of predicted root biomass vs. observation at the continental

96 level. Predictions at each continent are generated by random forest models. Random forest

97 models were trained by samples excluding observations of the corresponding continent. The red

98 line is the 1:1 line.

99 


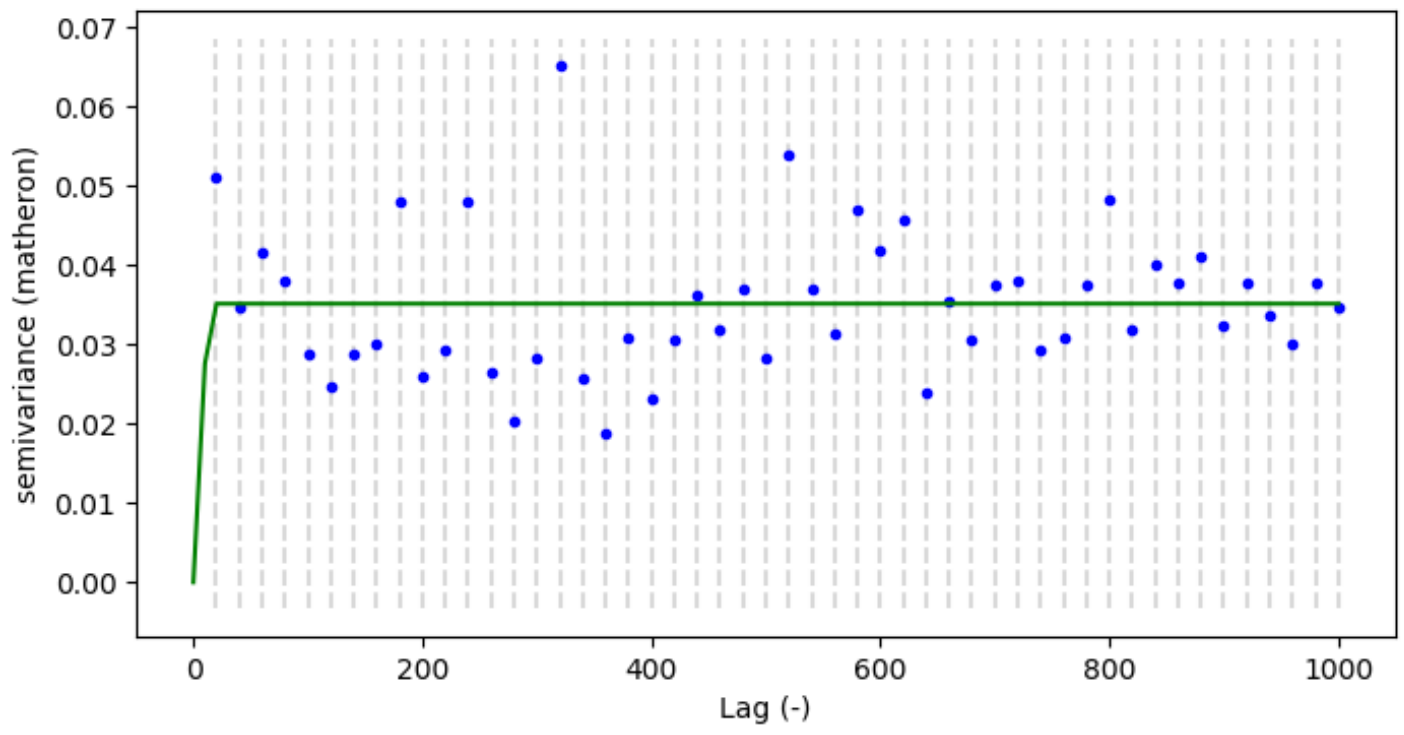

100

101

102

Supplementary Figure 10. Semivariogram of the random forest prediction errors.

103

104

105

106 

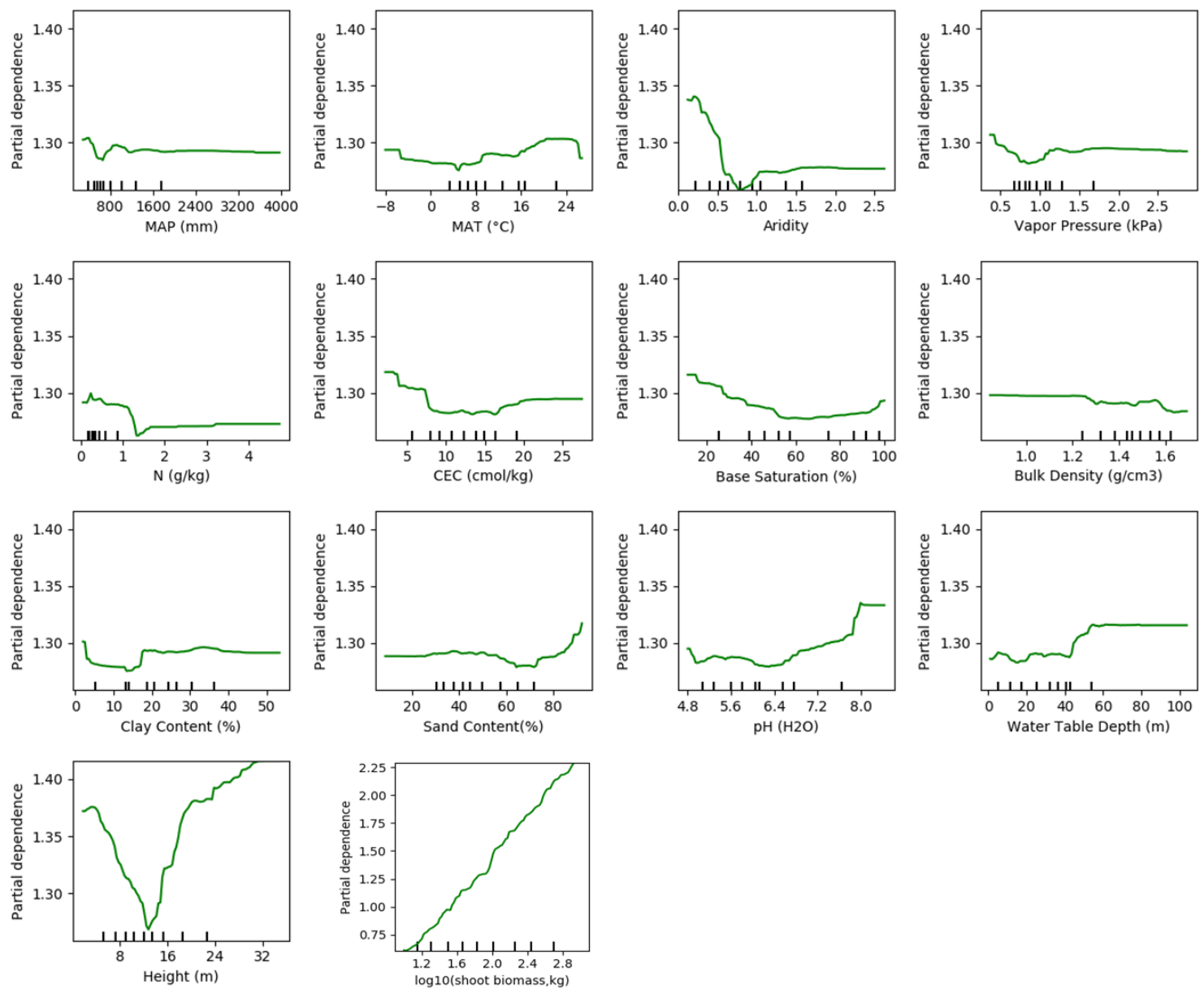

Supplementary Figure 11. Partial dependence plots showing the dependence of root biomass on predictors for woody plant with shoot biomass $>10 \mathrm{~kg}$. $10 \mathrm{~kg}$ is one threshold on which we split our datasets for the best model performance (see Methods). Note the y-axis of the last panel (shoot biomass) is different from other predictors.

113 

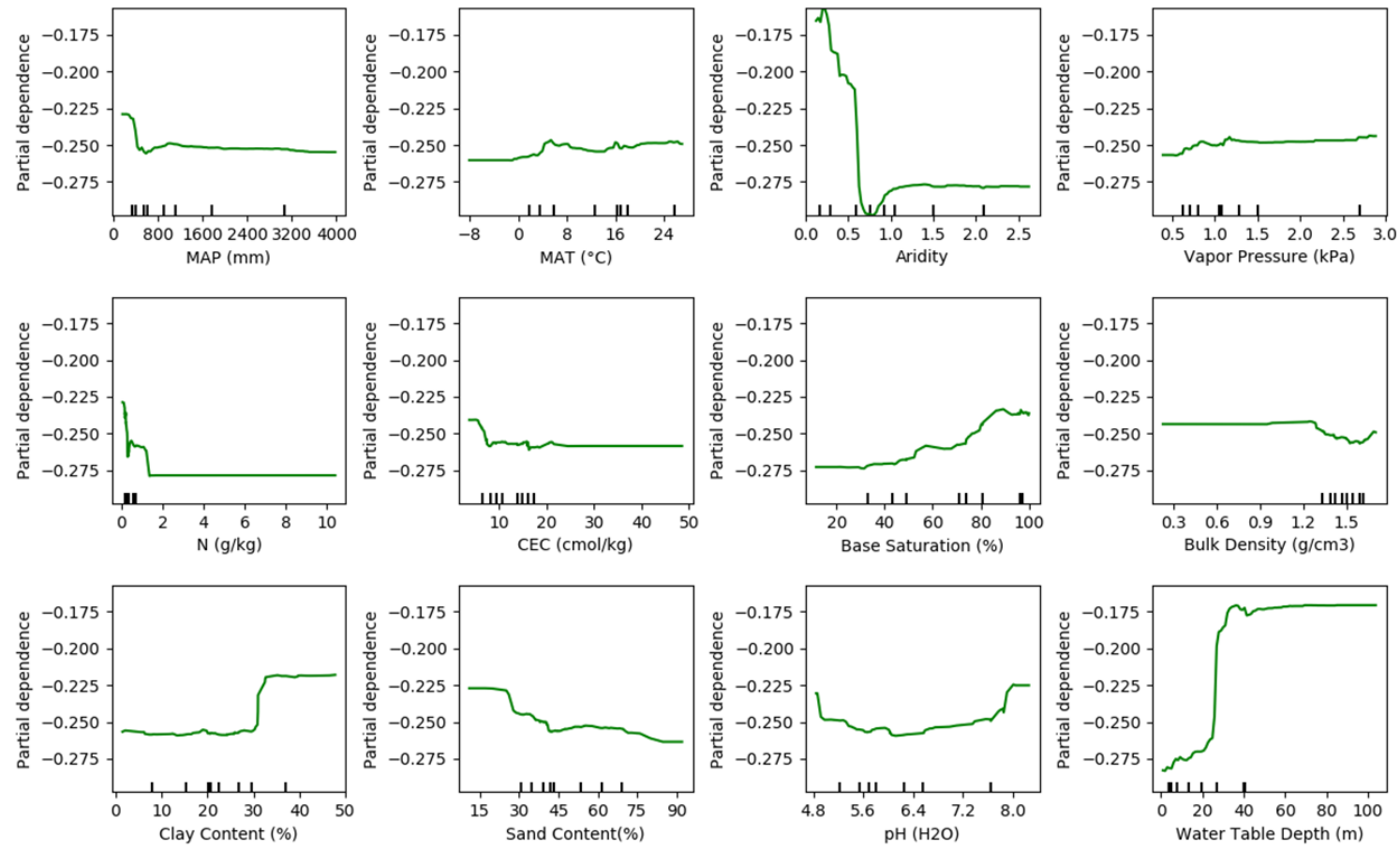

114
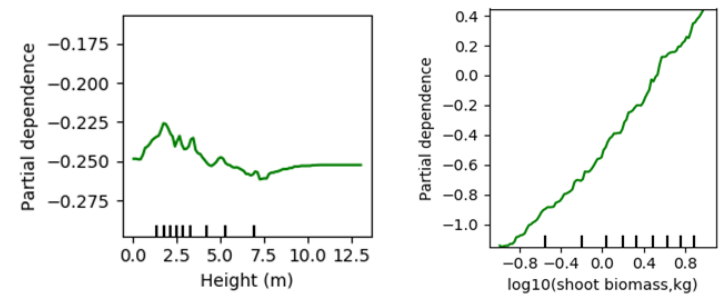

115

116

117

118

119

120

121

122

123

124

125

126

127

128

129

130

131

132

133

134
Supplementary Figure 12. Partial dependence plot showing the dependence of root biomass on predictors for woody plant with shoot biomass between [0.1 10] kg. 0.1 and $10 \mathrm{~kg}$ are thresholds on which we split our datasets for the best model performance (see Methods). Note the y-axis of the last panel (shoot biomass) is different from other predictors. 

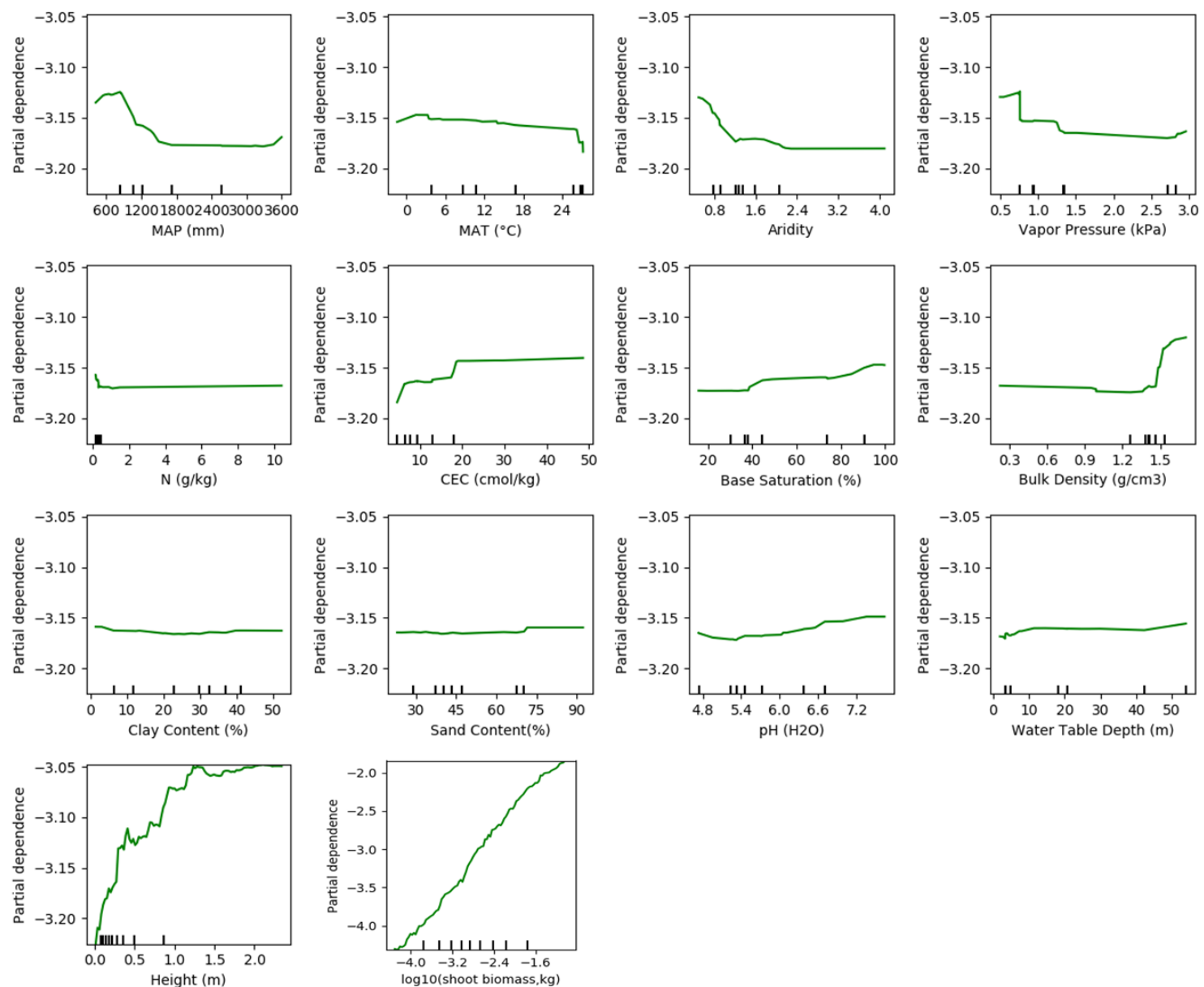

Supplementary Figure 13. Partial dependence plot showing the dependence of root biomass on predictors for woody plant with shoot biomass smaller than $0.1 \mathrm{~kg} .0 .1 \mathrm{~kg}$ is one threshold on which we split our datasets for the best model performance (see Methods). Note the y-axis of the last panel (shoot biomass) is different from other predictors. 
155 Supplementary Table 1. The source, unit, category, resolution, time coverage and reference of 156 gridded global datasets used in building training model and predicting root biomass. BIO2-11

157 and BIO13-19 corresponds to Bioclimatic variables from WorldClim version 2. All datasets were

158 accessed in February 2019.

\begin{tabular}{|c|c|c|c|c|c|c|}
\hline Name & Source & Unit & Type & Res & Time & Reference \\
\hline Age & Mixed & year & Biological & $1 \mathrm{~km}$ & Current & See Methods for details \\
\hline $\begin{array}{l}\text { Maximum } \\
\text { Rooting } \\
\text { Depth }\end{array}$ & GSES & $\mathrm{m}$ & Biological & $1 \mathrm{~km}$ & Current & $\begin{array}{l}\text { http://globalchange. bnu.edu.cn/research/s } \\
\text { oilw }\end{array}$ \\
\hline Biome & $\begin{array}{l}\text { The nature } \\
\text { conservancy }\end{array}$ & & Biological & $1 \mathrm{~km}$ & Current & http://maps.tnc.org/gis_data.html \\
\hline Height & Simard & $\mathrm{m}$ & Biological & $1 \mathrm{~km}$ & Current & $\begin{array}{l}\text { https://webmap.ornl.gov/wcsdown/dataset } \\
\text {.jsp?ds id=10023 }\end{array}$ \\
\hline $\begin{array}{l}\text { Aboveground } \\
\text { biomass } \\
\text { density }\end{array}$ & $\begin{array}{l}\text { GlobBiomas } \\
\text { s }\end{array}$ & $\mathrm{Mg} / \mathrm{ha}$ & Biological & $1 \mathrm{~km}$ & Current & $\begin{array}{l}\text { http://globbiomass.org/wp- } \\
\text { content/uploads/GB_Maps/Globbiomass } \\
\text { global_dataset.html }\end{array}$ \\
\hline Tree density & Crowther & per ha & Biological & $1 \mathrm{~km}$ & Current & $\begin{array}{l}\text { https://elischolar.library.yale.edu/yale_fes } \\
\text { data/1/ }\end{array}$ \\
\hline $\begin{array}{l}\text { Rooting } \\
\text { depth }\end{array}$ & Fan & $\mathrm{m}$ & Biological & & Current & $\begin{array}{l}\text { https://wci.earth2observe.eu/thredds/catal } \\
\text { og/usc/root-depth/catalog.html }\end{array}$ \\
\hline Bulk Density & GSES & $\mathrm{g} / \mathrm{cm}^{3}$ & Soil & $1 \mathrm{~km}$ & Current & $\begin{array}{l}\text { http://globalchange. bnu.edu.cn/research/s } \\
\text { oilw }\end{array}$ \\
\hline $\begin{array}{l}\text { Soil Organic } \\
\text { Matter }\end{array}$ & GSES & $\begin{array}{l}\% \text { of } \\
\text { weight }\end{array}$ & Edaphic & $1 \mathrm{~km}$ & Current & http://globalchange. bnu.edu.cn/research/s \\
\hline Soil pH & GSES & & Edaphic & $1 \mathrm{~km}$ & Current & $\frac{\text { http://globalchange. } b n u . e d u . c n / r e s e a r c h / s}{\text { oilw }}$ \\
\hline Soil Sand & GSES & $\begin{array}{l}\% \text { of } \\
\text { weight }\end{array}$ & Edaphic & $1 \mathrm{~km}$ & Current & http://globalchange.bnu.edu.cn/research/s \\
\hline Soil Clay & GSES & $\begin{array}{l}\% \text { of } \\
\text { weight }\end{array}$ & Edaphic & $1 \mathrm{~km}$ & Current & 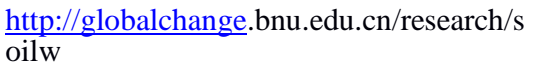 \\
\hline $\begin{array}{l}\text { Total } \\
\text { Nitrogen }\end{array}$ & GSES & $\begin{array}{l}\% \text { of } \\
\text { weight }\end{array}$ & Edaphic & $1 \mathrm{~km}$ & Current & 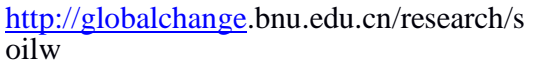 \\
\hline $\begin{array}{l}\text { Total } \\
\text { Phosphorus }\end{array}$ & GSES & $\begin{array}{l}\% \text { of } \\
\text { weight }\end{array}$ & Edaphic & $1 \mathrm{~km}$ & Current & 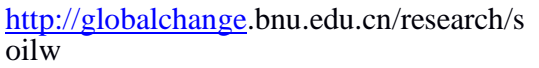 \\
\hline $\begin{array}{l}\text { Bray } \\
\text { Phosphorus }\end{array}$ & GSES & ppm & Edaphic & $1 \mathrm{~km}$ & Current & $\begin{array}{l}\text { http://globalchange. } \\
\text { oilw }\end{array}$ \\
\hline $\begin{array}{l}\text { Total } \\
\text { Potassium }\end{array}$ & GSES & $\begin{array}{l}\% \text { of } \\
\text { weight }\end{array}$ & Edaphic & $1 \mathrm{~km}$ & Current & $\begin{array}{l}\text { http://globalchange. bnu.edu.cn/research/s } \\
\text { oilw }\end{array}$ \\
\hline $\begin{array}{l}\text { Exchangeabl } \\
\text { e Aluminum }\end{array}$ & GSES & $\mathrm{cmol} / \mathrm{kg}$ & Edaphic & $1 \mathrm{~km}$ & Current & 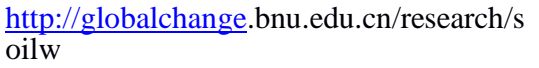 \\
\hline $\begin{array}{l}\text { Cation } \\
\text { Exchange }\end{array}$ & GSES & $\mathrm{cmol} / \mathrm{kg}$ & Edaphic & $1 \mathrm{~km}$ & Current & http://globalchange.. bnu.edu.cn/research/s \\
\hline $\begin{array}{l}\text { Capacity } \\
\text { Base } \\
\text { Saturation }\end{array}$ & GSES & $\%$ & Edaphic & $1 \mathrm{~km}$ & Current & $\frac{\text { http://globalchange. } b n u . e d u . c n / r e s e a r c h / s}{\text { oilw }}$ \\
\hline Soil Moisture & ESA CCI & $\mathrm{m} 3 / \mathrm{m} 3$ & Edaphic & $0.25^{\circ}$ & $\begin{array}{l}\text { Average } \\
1982- \\
2005\end{array}$ & https://www.esa-soilmoisture-cci.org/ \\
\hline $\begin{array}{l}\text { Water Table } \\
\text { Depth }\end{array}$ & Fan2013 & $\mathrm{m}$ & Edaphic & $1 \mathrm{~km}$ & Current & $\begin{array}{l}\text { https://glowasis.deltares.nl/thredds/catalo } \\
\text { g/opendap/opendap/Equilibrium_Water_ } \\
\text { Table/catalog.html }\end{array}$ \\
\hline $\begin{array}{l}\text { Mean Annual } \\
\text { Precipitation }\end{array}$ & $\begin{array}{l}\text { WorldClim } \\
\text { V2.0 }\end{array}$ & $\mathrm{mm}$ & Climatic & $1 \mathrm{~km}$ & $\begin{array}{l}\text { Average } \\
1970- \\
2000\end{array}$ & http://www.worldclim.org \\
\hline $\begin{array}{l}\text { Mean Annual } \\
\text { Temperature }\end{array}$ & $\begin{array}{l}\text { WorldClim } \\
\text { V2.0 }\end{array}$ & ${ }^{\circ} \mathrm{C}$ & Climatic & $1 \mathrm{~km}$ & $\begin{array}{l}\text { Average } \\
1970- \\
2000\end{array}$ & http://www.worldclim.org \\
\hline Aridity & GA-ET & & Climatic & $1 \mathrm{~km}$ & $\begin{array}{l}\text { Average } \\
1970- \\
2000\end{array}$ & $\begin{array}{l}\text { https://figshare.com/articles/Global_Aridi } \\
\text { ty } \\
\text { _Index_and_Potential_Evapotranspiration } \\
\text { _ET0_Climate_Database_v2/7504448/3 }\end{array}$ \\
\hline $\begin{array}{l}\text { Potential } \\
\text { Evapotranspi } \\
\text { ration }\end{array}$ & GA-ET & $\mathrm{mm}$ & Climatic & $1 \mathrm{~km}$ & $\begin{array}{l}\text { Average } \\
1970- \\
2000\end{array}$ & $\begin{array}{l}\text { https://figshare.com/articles/Global_Aridi } \\
\text { ty } \\
\text {-Index_and_Potential_Evapotranspiration } \\
\text { ET0_Climate_Database_v2/7504448/3 }\end{array}$ \\
\hline $\begin{array}{l}\text { Solar } \\
\text { Radiation }\end{array}$ & $\begin{array}{l}\text { WorldClim } \\
\text { V2.0 }\end{array}$ & $\begin{array}{l}\mathrm{kJ} / \mathrm{m} 2 \\
/ \text { day }\end{array}$ & Climatic & $1 \mathrm{~km}$ & $\begin{array}{l}\text { Average } \\
1970- \\
2000\end{array}$ & http://www.worldclim.org \\
\hline Vapor & WorldClim & $\mathrm{kPa}$ & Climatic & $1 \mathrm{~km}$ & Average & http://www.worldclim.org \\
\hline
\end{tabular}




\begin{tabular}{|c|c|c|c|c|c|c|}
\hline Pressure & V2.0 & & & & $\begin{array}{l}1970- \\
2000\end{array}$ & \\
\hline $\begin{array}{l}\text { Cumulative } \\
\text { Water Deficit }\end{array}$ & $\begin{array}{l}\text { WorldClim } \\
\text { V2.0 }\end{array}$ & $\mathrm{mm}$ & Climatic & $1 \mathrm{~km}$ & $\begin{array}{l}\text { Average } \\
1970- \\
2000\end{array}$ & PET - MAP \\
\hline Wind Speed & $\begin{array}{l}\text { WorldClim } \\
\text { V2.0 }\end{array}$ & $\mathrm{m} / \mathrm{s}$ & Climatic & $1 \mathrm{~km}$ & $\begin{array}{l}\text { Average } \\
1970- \\
2000\end{array}$ & http://www.worldclim.org \\
\hline BIO2-11 & $\begin{array}{l}\text { WorldClim } \\
\text { V2.0 }\end{array}$ & & Climatic & $1 \mathrm{~km}$ & $\begin{array}{l}\text { Average } \\
1970- \\
2000\end{array}$ & http://www.worldclim.org \\
\hline BIO13-19 & $\begin{array}{l}\text { WorldClim } \\
\text { V2.0 }\end{array}$ & & Climatic & $1 \mathrm{~km}$ & $\begin{array}{l}\text { Average } \\
1970- \\
2000\end{array}$ & http://www.worldclim.org \\
\hline Elevation & $\begin{array}{l}\text { SRTM30_P } \\
\text { LUS v8 }\end{array}$ & $\mathrm{m}$ & $\begin{array}{l}\text { Topograph } \\
\text { ical }\end{array}$ & $1 \mathrm{~km}$ & $\begin{array}{l}\text { Average } \\
1970- \\
2000\end{array}$ & $\begin{array}{l}\text { https://eatlas.org.au/data/uuid/80301676 } \\
\text { 97fb-4bdf-b06c-e961e5c0cb0b }\end{array}$ \\
\hline
\end{tabular}

161 Supplementary Table 2. Alterative global datasets for quantifying root biomass prediction 162 uncertainty. All datasets were accessed in June 2019.

\begin{tabular}{|c|c|c|c|c|}
\hline Name & Variables & Res & Time & Reference \\
\hline AGB_Hu & Shoot biomass & $1 \mathrm{~km}$ & Current & Hu, et al. ${ }^{2}$ \\
\hline AGB_Liu & Shoot biomass & $0.25^{\circ}$ & $1993-2012$ & Liu, et al. ${ }^{3}$ \\
\hline AGB_GeoC & Shoot biomass & 0.01 & Current & $\begin{array}{l}\text { GEOCARBON, https://www.bgc- } \\
\text { jena.mpg.de/geodb/projects/Home.php }\end{array}$ \\
\hline SoilGrid & $\begin{array}{l}\text { CEC, Bulk density, Clay } \\
\text { content, Sand content, CEC, }\end{array}$ & $1 \mathrm{~km}$ & Current & Hengl, et al. ${ }^{4}$ \\
\hline WISE30 & $\begin{array}{l}\text { Total nitrogen, } \mathrm{pH} \text {, Bulk } \\
\text { density, clay, sand, Base } \\
\text { saturation, CEC, }\end{array}$ & $1 \mathrm{~km}$ & Current & Batjes ${ }^{5}$ \\
\hline CHELSA & MAT & $1 \mathrm{~km}$ & Same as WorldClim & http://chelsa-climate.org/ \\
\hline TerraClimate & Aridity, MAP, Vapor pressure & $4 \mathrm{~km}$ & Same as WorldClim & $\begin{array}{l}\text { http://www.climatologylab.org/terracli } \\
\text { mate.html }\end{array}$ \\
\hline CRU_TS4.03 & $\begin{array}{l}\text { Vapor pressure, MAP, MAT, } \\
\text { aridity }\end{array}$ & $0.5^{\circ}$ & Same as WorldClim & https://crudata.uea.ac.uk/cru/data/hrg/ \\
\hline
\end{tabular}

164 Supplementary Table 3. Land area, land area occupied by woody plants (forest area), shoot 165 biomass, root biomass and weighted $R: S$ ratio (total shoot biomass/total root biomass) at the 166 biome and global scales. The biome classification is from The Nature Conservancy ${ }^{1}$. Forest area 167 covers land with canopy cover $>15 \%^{6}$. Numbers after \pm are $95 \%$ confidence intervals (see

168 Methods).

\begin{tabular}{|c|c|c|c|c|c|c|}
\hline $\begin{array}{l}\text { Biome } \\
\text { number }\end{array}$ & Name & $\begin{array}{l}\text { Land area } \\
\left(10^{6} \mathrm{~km}^{2}\right)\end{array}$ & $\begin{array}{l}\text { Forest area } \\
\left(10^{6} \mathbf{k m}^{2}\right)\end{array}$ & $\begin{array}{l}\text { Shoot biomass } \\
\text { (Pg) }\end{array}$ & $\begin{array}{l}\text { Root biomass } \\
\text { (Pg) }\end{array}$ & $\begin{array}{l}\text { Weighted } R: S \\
\text { Ratio }\end{array}$ \\
\hline 1 & Tropical moist & 19.8 & 15.6 & 295 & $71.7 \pm 23$ & $0.24 \pm 0.08$ \\
\hline 2 & Boreal & 16 & 11.2 & 77.5 & $19.5 \pm 6.5$ & $0.25 \pm 0.08$ \\
\hline 3 & Tropical savanna & 19.5 & 6.7 & 52 & $13.7 \pm 3$ & $0.26 \pm 0.06$ \\
\hline 4 & Temperate broadleaf & 12.9 & 5.8 & 66 & $16.6 \pm 4.6$ & $0.25 \pm 0.07$ \\
\hline 5 & Temperate coniferous & 4.4 & 2.5 & 32.2 & $8.2 \pm 2.1$ & $0.25 \pm 0.07$ \\
\hline 6 & Tropical dry & 3.8 & 1.4 & 13.7 & $3.8 \pm 4.2$ & $0.28 \pm 0.31$ \\
\hline 7 & Tundra & 8.0 & 0.9 & 3.9 & $1.1 \pm 0.7$ & $0.28 \pm 0.18$ \\
\hline 8 & Temperate savanna & 9.6 & 0.7 & 4.7 & $1.4 \pm 0.7$ & $0.30 \pm 0.15$ \\
\hline 9 & Montane & 5.2 & 0.5 & 4.3 & $1.3 \pm 1.1$ & $0.30 \pm 0.26$ \\
\hline 10 & Mediterranean & 3.3 & 0.5 & 4.8 & $1.5 \pm 0.7$ & $0.31 \pm 0.15$ \\
\hline
\end{tabular}




\begin{tabular}{lllllll}
\hline 11 & Tropical coniferous & 0.6 & 0.4 & 3.3 & $0.9 \pm 0.4$ & $0.27 \pm 0.12$ \\
12 & Desert & 27.9 & 0.4 & 2.9 & $0.9 \pm 0.6$ & $0.31 \pm 0.21$ \\
13 & Flooded savanna & 1.1 & 0.3 & 2 & $0.5 \pm 0.4$ & $0.25 \pm 0.18$ \\
14 & Mangroves & 0.3 & 0.2 & 2.1 & $0.4 \pm 0.2$ & $0.19 \pm 0.10$ \\
& Globe & 132.4 & 47.3 & 566.2 & $141.6 \pm 25.1$ & $0.25 \pm 0.04$ \\
\hline
\end{tabular}

171 Supplementary Table 4. Mean and median $R: S$ from observations and predicted in this study.

172 The mean $R: S$ is the arithmetic average of individual $\mathrm{R}: \mathrm{S}$ across site level observations (Obs) or 173 gridcells (Gridded). The median is the $50^{\text {th }}$ percentile across observations (Obs) or gridcells 174 (Girdded). Note the mean and median $R: S$ are different from the weighted $R: S$ from the last 175 column of Table 3 which shows the ratio between total root biomass and shoot biomass. The weighted $R: S$ is weighted by biomass while the mean and median are not weighted by biomass.

\begin{tabular}{llllll}
\hline $\begin{array}{l}\text { Biome } \\
\text { number }\end{array}$ & Name & $\begin{array}{l}\text { Mean } \\
(\text { Obs })\end{array}$ & $\begin{array}{l}\text { Median } \\
(\text { Obs })\end{array}$ & $\begin{array}{l}\text { Mean } \\
\text { (Gridded) }\end{array}$ & $\begin{array}{l}\text { Median } \\
\text { (Gridded) }\end{array}$ \\
\hline 1 & Tropical moist & 0.37 & 0.32 & 0.26 & 0.24 \\
2 & Boreal & 0.45 & 0.32 & 0.27 & 0.26 \\
3 & Tropical savanna & 0.44 & 0.36 & 0.29 & 0.27 \\
4 & Temperate broadleaf & 0.58 & 0.38 & 0.28 & 0.26 \\
5 & Temperate coniferous & 0.29 & 0.25 & 0.29 & 0.26 \\
6 & Tropical dry & & & 0.33 & 0.30 \\
7 & Tundra & & & 0.34 & 0.29 \\
8 & Temperate savanna & 0.74 & 0.45 & 0.36 & 0.33 \\
9 & Montane & 0.42 & 0.42 & 0.41 & 0.35 \\
10 & Mediterranean & 0.43 & 0.35 & 0.39 & 0.35 \\
11 & Tropical coniferous & 0.67 & 0.55 & 0.35 & 0.31 \\
12 & Desert & & & 0.40 & 0.35 \\
13 & Flooded savanna & & & 0.33 & 0.32 \\
14 & Mangroves & 0.47 & 0.40 & 0.26 & 0.25 \\
& Globe & 0.50 & 0.36 & 0.29 & 0.26 \\
\hline
\end{tabular}

\section{Comparison with published results}

181 There are few studies quantifying large scale vegetation root biomass. We searched

182 through the literature and compared our study with earlier studies ${ }^{7-10}$. We grouped here forests

183 into mega-biomes of tropical, temperate and boreal systems to enable a comparison between

184 different studies that used different forest biome definitions and areas (see Table 5). The three

185 mega-biomes together hold $\sim 68 \%$ of the global total root biomass ${ }^{7}$ (forest and non-forest

186 together), and are also commonly reported and therefore convenient to compare across studies. It 
187 is unclear whether forest in tropical/subtropical grasslands, savannas and shrublands (Biome 3,

188 Supplementary Figure 2) should be treated as a tropical forest across studies. Similarly, it is 189 unclear whether forest in temperate grasslands/savannas and shrublands (Biome 8) should be 190 treated as a temperate forest, and forest in tundra (Biome 7) as a boreal forest. We therefore 191 conducted two series of comparisons with and without the above-mentioned ambiguous forest 192 classes. In series 1 (S1), Biomes 1, 6, 11 and 3 (Biome distribution is displayed in

193 Supplementary Figure 2) are aggregated to represent tropical systems; Biomes 3, 5, 8 are 194 grouped into temperate forest; and Biomes 6 and 7 are grouped into boreal forest. In series 2 195 (S2), we grouped Biomes 1,2,3 into tropical forest, Biomes 4 and 5 into temperate forest and 196 Biomes 6 as boreal forest. Together, root biomass from tropical, temperate and boreal forests is 197 44-183\% higher in earlier studies than in S1 and 65-226\% higher than in S2 (Table 5).

This over-estimation from earlier studies is largely explained by an over-estimation of

199 shoot biomass by earlier studies. To demonstrate this, we compiled additional studies (Table 6)

200 that reported shoot biomass at the global, tropical, temperate and boreal forests.

201 The global forest root biomass ranges between $154-210 \mathrm{Pg}$ if root biomass was 202 upscaled through different allometric equations collected from literature (Table 7). A prediction 203 of root biomass after fitting our site-level data with an allometric equation (fitted equation: $R=$ $2040.289 S^{0.974}, R^{2}=0.79$, Table 7) yielded a global forest root biomass of $155 \mathrm{Pg}$ (tree-level-

205 upscaling) or $172 \mathrm{Pg}$ (stand-level-upscaling), which is larger than $147 \mathrm{Pg}$ from the RF up-scaling 206 model. For stand-level-upscaling, we followed the practice in literature ${ }^{11,12}$ and assumed an 207 allometric equation is equally applicable to stand level data (weight per area) despite being 208 derived from individual-level data. Root biomass density (weight per area) was directly 209 estimated from GlobBiomass-AGB ${ }^{13}$ shoot biomass density through the allometric equations. In 210 tree-level upscaling, similarly to the RF upscaling procedure, GlobBiomass-AGB ${ }^{13}$ shoot

211 biomass density was firstly downscaled to individual tree level through tree density ${ }^{14}$. Allometric

212 equations were applied to estimate tree level root biomass (weight per plant), which is then

213 transferred into per area level through the same tree density. Whether it is upscaled from the

214 individual-tree-level or the stand-level is unlikely to explain the overestimation as there is no 215 systematic difference between these two approaches (Table 7).

217 Supplementary Table 5. Comparison between studies quantifying root biomass in tropical, 
218 temperate and boreal forests. This table expands upon Table 1 in the main text with shoot 219 biomass, land area, biomass density and $R: S$.

\begin{tabular}{|c|c|c|c|c|c|c|}
\hline & & This study ${ }^{\text {S1 }}$ & This study ${ }^{\text {S2 }}$ & Jackson $1997^{7}$ & Saugier2001 ${ }^{15}$ & Robinson $2007^{10}$ \\
\hline Method & & $\begin{array}{l}\text { Machine } \\
\text { learning }\end{array}$ & $\begin{array}{l}\text { Machine } \\
\text { learning }\end{array}$ & $\begin{array}{l}\text { Biome } \\
\text { average root } \\
\text { biomass } \\
\text { density, area }\end{array}$ & $\begin{array}{l}\text { Biome average } \\
R: S \text { ratio, shoot } \\
\text { biomass density, } \\
\text { area }\end{array}$ & $\begin{array}{l}\text { Biome average } \\
R: S \text { ratio, shoot } \\
\text { biomass density, } \\
\text { area }\end{array}$ \\
\hline Root & Tropical (Tr, Pg) & 92 & 76 & 114 & 147 & 246 \\
\hline \multirow[t]{5}{*}{ biomass } & Temperate $(\mathrm{Te}, \mathrm{Pg})$ & 26 & 25 & 51 & 59 & 98 \\
\hline & Boreal $(\mathrm{Bo}, \mathrm{Pg})$ & 21 & 20 & 35 & 30 & 50 \\
\hline & $\mathrm{Tr}+\mathrm{Te}+\mathrm{Bo}(\mathrm{Pg})$ & 139 & 121 & 200 & 236 & 394 \\
\hline & $\mathrm{RD}_{\mathrm{S} 1}{ }^{*}$ & $0 \%$ & & $44 \%$ & $70 \%$ & $183 \%$ \\
\hline & $\mathrm{RD}_{\mathrm{S} 2}{ }^{\&}$ & & $0 \%$ & $65 \%$ & $95 \%$ & $226 \%$ \\
\hline Shoot & Tropical & 364 & 312 & & 532 & 532 \\
\hline biomass & Temperate & 102.9 & 98.2 & & 218.4 & 218.4 \\
\hline$(\mathrm{Pg})$ & Boreal & 81.4 & 77.5 & & 83.6 & 83.6 \\
\hline Forest & Tropical & 24.1 & 17.4 & 24.5 & 17.5 & 17.5 \\
\hline area & Temperate & 9 & 8.3 & 12 & 10.4 & 10.4 \\
\hline $\begin{array}{c}\left(10^{6}\right. \\
\left.\mathrm{km}^{2}\right)\end{array}$ & Boreal & 12.1 & 11.2 & 12 & 13.7 & 11.2 \\
\hline Root & Tropical & 3.8 & 4.4 & 4.6 & 8.4 & 14.0 \\
\hline density & Temperate & 2.9 & 3.0 & 4.2 & 5.7 & 9.4 \\
\hline$(\mathrm{kg} / \mathrm{m} 2)$ & Boreal & 1.7 & 1.8 & 2.9 & 2.2 & 4.5 \\
\hline Shoot & Tropical & 15.1 & 17.9 & & 30.4 & 30.4 \\
\hline density & Temperate & 11.4 & 11.8 & & 21 & 21 \\
\hline \multirow[t]{2}{*}{$(\mathrm{kg} / \mathrm{m} 2)$} & Boreal & 6.73 & 6.9 & & 6.1 & 7.5 \\
\hline & Tropical & 0.25 & 0.24 & & 0.28 & 0.46 \\
\hline Average & Temperate & 0.25 & 0.25 & & 0.26 & 0.45 \\
\hline$R: S$ & Boreal & 0.26 & 0.26 & & 0.37 & 0.6 \\
\hline
\end{tabular}

$\mathrm{S} 1$. Tropical moist forest (Biome 1), tropical dry forest (Biome 6), tropical/subtropical coniferous forest (Biome 11) and forest in tropical/subtropical grasslands/savannas and shrublands (Biome 3) are aggregated to represent tropical systems (Tr). Temperate broadleaf/mixed forest (Biome 4), temperate coniferous forest (Biome 5) and forest in temperate grasslands/savannas and shrublands (Biome 8) are merged together as temperate systems (Te). Boreal forest (Biome 2) and woody plants in tundra region (Biome 7) are aggregated as boreal forest (Bo). Biome classification is from The Nature Conservancy ${ }^{1}$ and is shown in Supplementary Figure 2.

S2. Tropical systems (Tr): Biomes 1,6,11; Temperate systems (Te) : Biomes 4,5; Boreal systems (Bo) : Biome 2.

${ }^{*} \mathrm{RDs} 1$, the relative difference of $\mathrm{Tr}+\mathrm{Te}+\mathrm{Bo}$ between this study (S1) and previous quantifications. RDs1 $=($ previous study this study)/this study x 100\%. For example, in the column with the head Jackson, RD $1=(200-139) / 139 * 100 \%=44 \%$.

${ }^{\&} \mathrm{RD}_{\mathrm{s} 2}$, the same as RDs1, but with the $\mathrm{S} 2$ definition of tropical, temperate and boreal systems.

Supplementary Table 6. Comparison between shoot biomass used in this study ${ }^{13}$ and other 233 estimates for tropical, temperate, boreal forests and the globe.

\begin{tabular}{|c|c|c|c|c|c|c|c|c|}
\hline & & This study ${ }^{\mathrm{s} 1}$ & This study $^{\mathrm{s} 2}$ & Pan2011 ${ }^{16,17}$ & Saatchi ${ }^{11}$ & Liu2015 $^{3}$ & Bacchini2017 ${ }^{18}$ & Hu2016 ${ }^{2}$ \\
\hline \multicolumn{2}{|l|}{ Method } & $\begin{array}{l}\text { GlobBiomass- } \\
\text { AGB }\end{array}$ & $\begin{array}{l}\text { GlobBiomass- } \\
\text { AGB }\end{array}$ & Inventory & Satellite & $\begin{array}{l}\text { Satellite } \\
\text { VOD }\end{array}$ & Satellite & $\begin{array}{l}\text { Satellite } \\
\text { LiDAR }\end{array}$ \\
\hline Time & & Current & Current & Current & $\sim 2000$ & $\sim 2000$ & $\sim 2007 / 8$ & Current \\
\hline Shoot & Tropical & 364 & 312 & 410 & $346-424$ & $360-416$ & 318 & \\
\hline biomass & Temperate & 102.9 & 98.2 & 88 & & 74-132 & & \\
\hline \multirow[t]{2}{*}{$(\mathrm{Pg})$} & Boreal & 81.4 & 77.5 & 72.4 & & $48-78$ & & \\
\hline & Globe & 566 & 566 & & & & & 533 \\
\hline
\end{tabular}

S1. Tropical moist forest (Biome 1), tropical dry forest (Biome 6), tropical/subtropical coniferous forest (Biome 11) and forest in tropical/subtropical grasslands/savannas and shrublands (Biome 3) are aggregated to represent tropical systems (Tr). Temperate broadleaf/mixed forest (Biome 4), temperate coniferous forest (Biome 5) and forest in temperate grasslands/savannas and shrublands (Biome 8) are merged together as temperate systems (Te). Boreal forest (Biome 2) and woody plants in tundra region (Biome 7) are aggregated as boreal forest (Bo). Biome classification is from The Nature Conservancy ${ }^{1}$ and is shown in Supplementary Figure 2. 
Supplementary Table 7. Global forest root biomass estimated from allometric equations.

\begin{tabular}{cccccc}
\hline & Fit & Jiang $^{\mathbf{1 9}}$ & Niklas $^{\mathbf{2 0}}$ & Robinson $^{\mathbf{}}$ & Cairns $^{\mathbf{2 1}}$ \\
\hline$\alpha$ & 0.289 & 0.332 & 0.372 & 0.384 & 0.338 \\
$\beta$ & 0.974 & 0.920 & 0.924 & 0.954 & 0.926 \\
Global Total $^{\mathrm{t}}(\mathrm{Pg})$ & 155 & 165 & 186 & 199 & 167 \\
Global Total $^{\mathrm{s}}(\mathrm{Pg})$ & 172 & 154 & 176 & 210 & 161
\end{tabular}

Global Total ${ }^{\mathrm{s}}(\mathrm{Pg})$

Fit: Observed root $(R)$ and shoot $(S)$ biomass were fitted into an allometric equation, $R=\alpha S^{\beta}$ where $\alpha$ and $\beta$ are allometric coefficients.

Jiang, Niklas and Robinson: coefficients of the allometric equation were taken from corresponding literature.

t: tree-based estimation. GlobBiomass-AGB shoot biomass was firstly transferred to individual tree level through tree density. Tree level root biomass was estimated from the allometric equation and the derived tree level shoot biomass. Tree level root biomass was then transferred into per area level through tree density. This approach takes the similar procedure as the machine learning approach.

s: stand-based estimation. Per area root biomass was directly estimated from GlobBiomass-AGB shoot biomass through the allometric equation. This approach mimics practice in literature ${ }^{11,12}$.

\section{Preliminary estimation of fine root biomass}

255 Broadly speaking, leaf and fine root biomass are highly linked ${ }^{22} \cdot \operatorname{Ref}^{22}$ derived an relationship 256 between annual leaf biomass production and annual root biomass production (Table 1 of $\operatorname{Ref}^{22}$ ).

257 Assuming an annual turnover of leaves and fine roots, we approximate fine root biomass through 258 above mentioned relationship and leaf biomass. Leaf biomass is estimated through the remote 259 sensed leaf area index (LAI) ${ }^{23,24}$ and the observation-based leaf mass per area (or the inverse of 260 specific leaf area $)^{25}$. We apply two LAI datasets, the GIMMS3 $\mathrm{g}^{24}$ and the GlobMAP ${ }^{23}$. We 261 estimate the total global fine root biomass in forest (with 15\% canopy cover threshold as in the 262 main text) to be $6.7 \mathrm{Pg}$ (GIMMS3g) or $7.7 \mathrm{Pg}$ (GlobMAP). We acknowledge leaves and fine 263 roots may not be in sync ${ }^{26}$ temporally and/or locally. Our estimation here is preliminary and can be improved with a better understanding of fine roots in the future.

\section{Arithmetic mean $R: S$ is always larger than shoot-biomass weighted mean $R: S$}

267 The general form of the allometric equation is given by:

$$
R / S=\alpha S^{\beta-1}
$$

271 mean $R: S$ is always larger than the biomass weighted mean. Suppose that we have two classes of 272 trees or forest stands that differ in shoot biomass, one with size $x$, and the other is $y$. We assume 273 the number of $x$ is $m$ if we look at the individual-tree-level, or the area is $m$ if we look at the 
274 stand or larger level, and $n$ is the number or area of $y$.

275 The (shoot) biomass weighted mean $R: S$ is:

$$
\frac{\alpha m x^{\beta}+\alpha n y^{\beta}}{m x+n y}
$$

278 The arithmetic mean $R: S$ is:

$$
\frac{\alpha m x^{\beta-1}+\alpha n y^{\beta-1}}{m+n}
$$

The difference between the weighted and arithmetic mean is:

$$
\text { deltaMean }=\frac{\alpha m x^{\beta}+\alpha n y^{\beta}}{m x+n y}-\frac{\alpha m x^{\beta-1}+\alpha n y^{\beta-1}}{m+n}
$$

By algebraic transformations, this equation can be transformed into:

$$
\text { deltaMean }=\frac{\alpha m n}{(m+n)(m x+n y)}(x-y)\left(x^{\beta-1}-y^{\beta-1}\right)
$$

Since we have $\alpha, m, n, x, y>0$, Equation SI2 tells if $\beta=1$, deltaMean $=0$; if $\beta<$ that the arithmetic mean $R: S$ always overestimate the (shoot) biomass weighted mean $R: S$.

\section{Allometric upscaling overestimates $R: S$ at $1 \mathrm{~km}$ resolution}

If we assume root and shoot biomass follow a universal allometric equation at different scales (Equation SI1), we show here we would always overestimate root biomass from the average shoot biomass at the pixel level. Here, we take the 1-km resolution as an example and upscaling to other resolutions follow the same logic. We start from upscaling from individual

293 trees and discuss later the case for the stand-level. Suppose we have two classes of trees or forest

294 stands that differ in shoot biomass, one with size $x$, and the other is $y$. In tropical forest, the

295 number of individuals $(N)$ generally follows a tight power law distribution, with the dominant

296 power function of the form $d^{-(\theta+1)}$, where $d$ is the tree diameter and $\theta$ is related to the

297 allometric exponent of the crown area to diameter ${ }^{29}$, which is relatively consistent across tropical

298 forests. Reported value of $\theta$ is around 1.27-1.31. In temperate or boreal forests, sometimes there

299 may lack the above power law size structure, and we will discuss this case later. The relationship

300 between tree diameter and biomass is highly conserved, with idealized trees exhibiting a general 301 allometric function where $A G B \propto d^{\omega}{ }^{30}$. The range of $\omega$ is between 1.1 and 3.37 from China's 
302 tree biomass equation database which consists of 5,924 biomass component equations for nearly

303200 species. Together,

$$
N=\mu A G B^{-\frac{\theta+1}{\omega}}
$$

305 where $\mu$ is a parameter with a positive value. We use $\gamma$ to replace $\frac{\theta+1}{\omega}$ for simplicity, and can 306 write

$$
N=\mu A G B^{-\gamma}
$$

308 The real $R: S$ ratio is,

$$
R S_{\text {real }}=\frac{\alpha \mu x^{\beta-\gamma}+\alpha \mu y^{\beta-\gamma}}{\mu x^{1-\gamma}+\mu y^{1-\gamma}}
$$

310 Which is the same as:

$$
R S_{r e a l}=\frac{\alpha\left(x^{\beta-\gamma}+y^{\beta-\gamma}\right)}{x^{1-\gamma}+y^{1-\gamma}}
$$

312 The estimated $R: S$ is:

$$
R S_{e s t i}=\alpha\left(\frac{\mu x^{1-\gamma}+\mu y^{1-\gamma}}{\mu x^{-\gamma}+\mu y^{-\gamma}}\right)^{\beta-1}
$$

314 Which is the same as:

$$
R S_{e s t i}=\alpha\left(\frac{x^{1-\gamma}+y^{1-\gamma}}{x^{-\gamma}+y^{-\gamma}}\right)^{\beta-1}
$$

316 Therefore, the difference between estimated and real $R: S$ is,

$$
\text { deltaRS }=R S_{\text {esti }}-R S_{\text {real }}=\alpha\left(\frac{\mu x^{1-\gamma}+\mu y^{1-\gamma}}{\mu x^{-\gamma}+\mu y^{-\gamma}}\right)^{\beta-1}-\frac{\alpha\left(x^{\beta-\gamma}+y^{\beta-\gamma}\right)}{x^{1-\gamma}+y^{1-\gamma}}
$$

318 With the condition $\beta<1, \alpha>0, \mu>0, x>0, y>0, \gamma>0$, deltaRS is always bigger than 0 , 319 as shown in Supplementary Figures 14, 15 numerically.

$320 \quad$ For forests without the power law structure or when we upscale from the stand-level measurement, we use $m$ and $n$ to denote the number of trees or the area of stands with the size of

322 shoot biomass $x$ and $y$.

323 The difference between estimated and real $R: S$ is,

$$
\text { deltaRS }=R S_{\text {esti }}-R S_{\text {real }}=\alpha\left(\frac{m x+n y}{m+n}\right)^{\beta-1}-\frac{\alpha\left(m x^{\beta}+n y^{\beta}\right)}{m x+n y}
$$

325 With the condition $\beta<1, \alpha>0, \mu>0, x>0, y>0, m>0, n>0, \gamma>0$, deltaRS is

326 always bigger than 0 as illustrated in Supplementary Figures 16, 17 numerically. 
328 The magnitude of overestimation is related to $\beta, \alpha, \mu, x, y, m, n$ (or $\gamma$ in case of forests with 329 power law size structure).

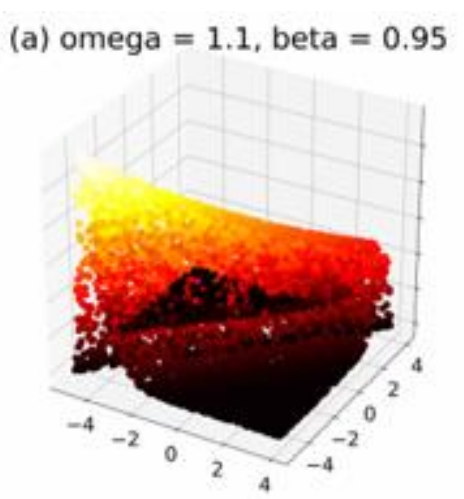

(c) omega $=1.1$, beta $=0.95$

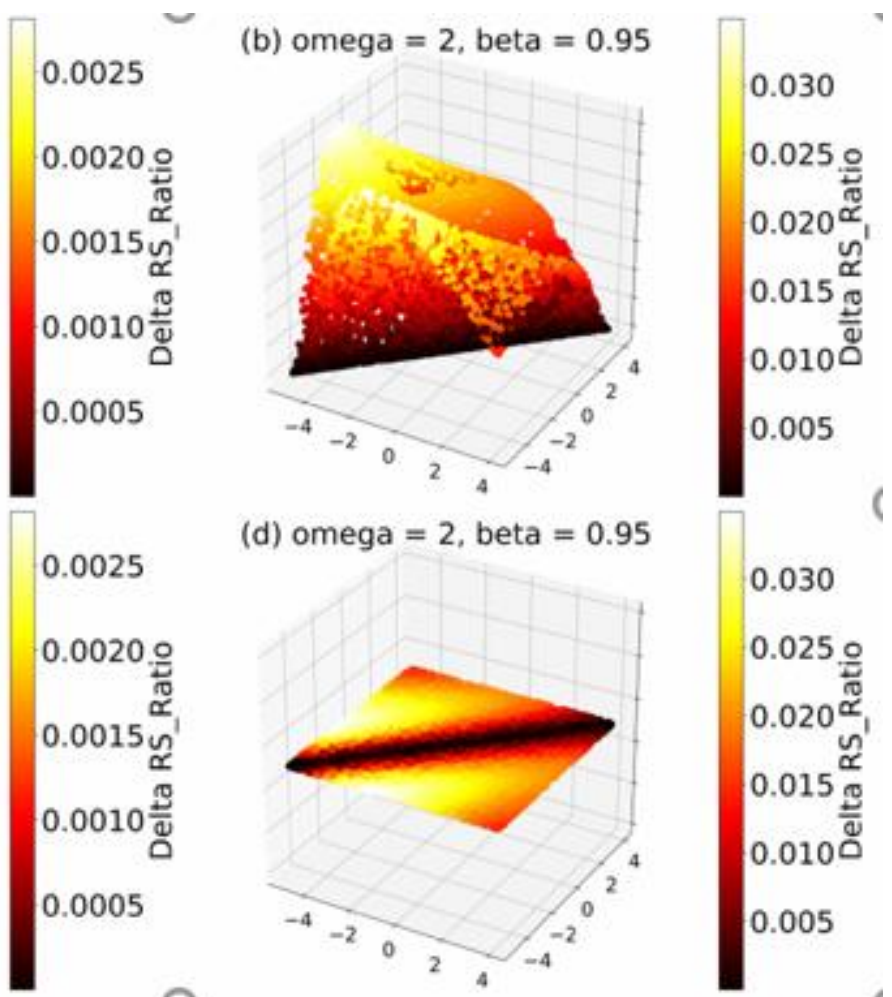

Supplementary Figure 14, deltaRS in responses to changes in tree sizes in $x$ ( $x$-axis) and $y(y$ axis). Size $x$ and size $y$ are randomly chosen with $\log x, \log y \in[-5,4]$. Here we fix $\alpha$ and $\theta$ with typical values $\quad \alpha=0.31, \theta=1.3$. (a) and (c) show deltaRS with $\omega=1.1, \beta=0.95$. (b) and (d) show deltaRS with $\omega=2, \beta=0.95$. (a) and (b) display deltaRS in a 3-dimentional space and the (c) and (d) are corresponding projections into the $x$ - $y$ space. deltaRS is always bigger than 0 with different values of $x, y, \alpha, \theta, \omega, \beta$ in literature. We choose fixed values for demonstration purpose here. See Equation SI3 for details. 

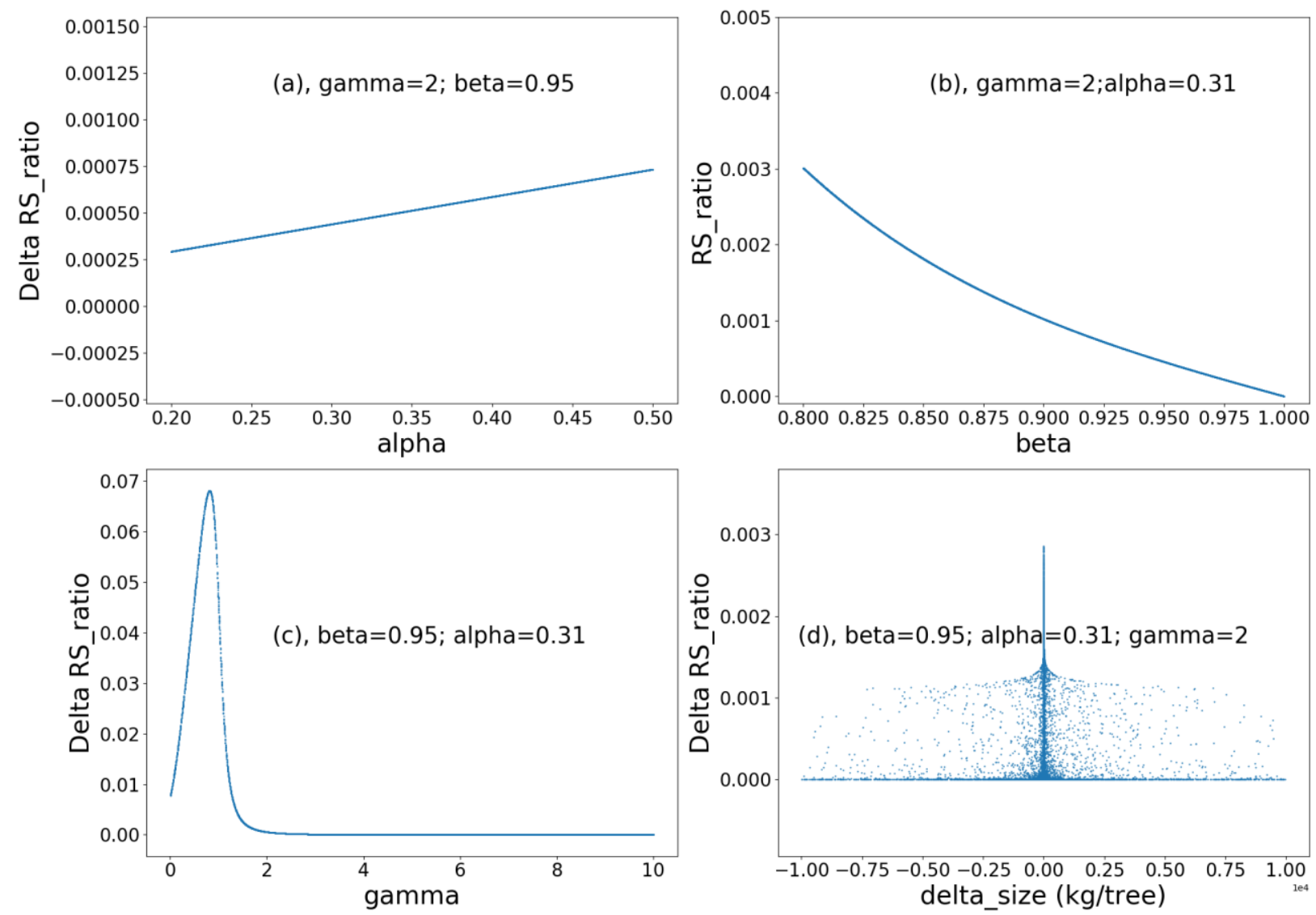

Supplementary Figure 15. deltaRS in responses to changes in $\alpha$ (a, alpha), $\beta$ (b, beta), $\gamma$ (c, gamma) and difference in tree size (d, delta_size). In panels (a), (b) and (c), the parameter in $x$ axis varies in a range that is broader than typically reported in literature while other parameters are fixed at a typical value. Panel (d) shows changes in deltaRS in response to differences in size $x$ and size $y$ where size $x$ and size $y$ are randomly generated with a uniform distribution of $\log x$ and $\log y$ with $\log x, \log y \in[-5,4]$. Note, in (d) Delta RS_ratio $=0$ when delta_size $=0$, but 


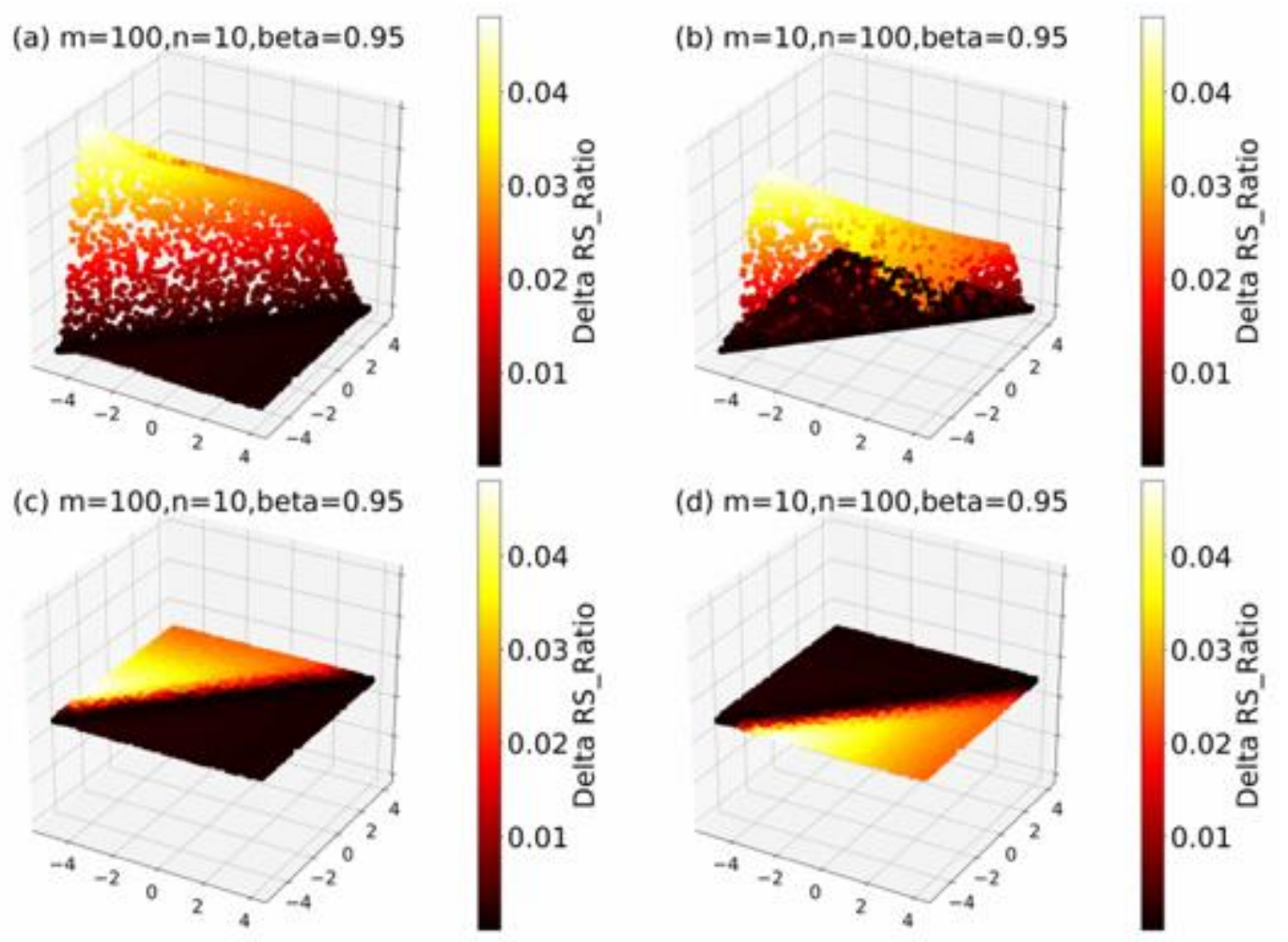

Supplementary Figure 16. deltaRS in responses to changes in tree sizes in $x$ ( $x$-axis) and $y(y$ axis). Size $x$ and size $y$ are randomly chosen with $\log x, \log y \in[-5,4]$. Here we fix $\alpha$ and $\theta$ with typical values $\alpha=0.31, \theta=1.3$. (a) and (c) show deltaRS with $m=100, n=10, \beta=0.95$. (b) and (d) show deltaRS with $m=10, n=100, \beta=0.95$. (a) and (b) display deltaRS in a 3dimentional space and (c) and (d) are their corresponding projection into the $x$ - $y$ space. deltaRS is always bigger than 0 with different values of $x, y, \alpha, \theta, m, n, \beta$ in literature. We choose fixed values for demonstration purpose here. See Equation SI4 for details. 

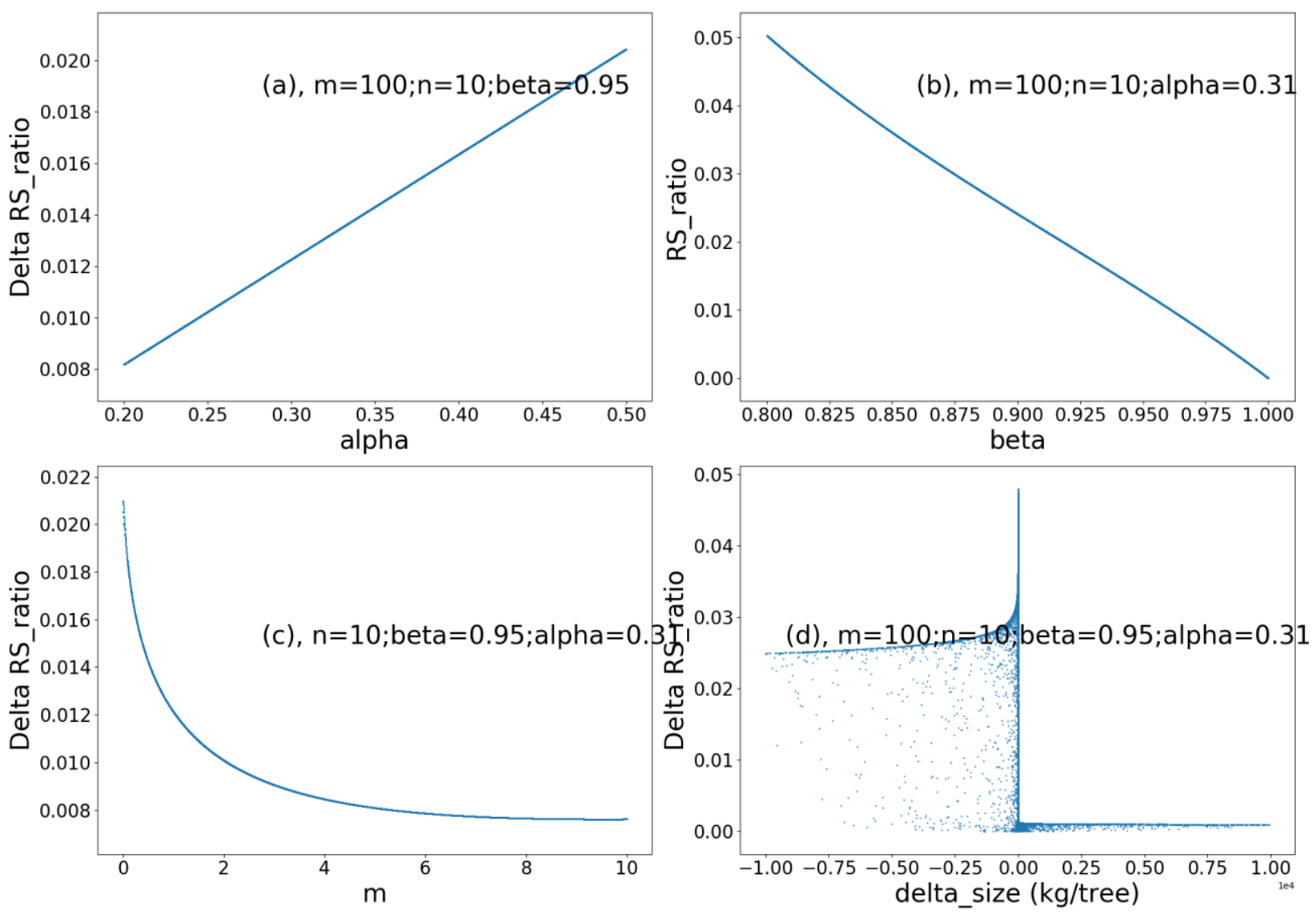

Supplementary Figure 17. deltaRS in responses to changes in $\alpha$ (a, alpha), $\beta$ (b, beta), number of trees or stand area of shoot biomass class $x(\mathrm{c}, m)$ and difference in tree size (d, delta_size). This figure is the same as Figure 15 except the exponent controlling the number of trees $(\gamma)$ is replaced by the number of trees or stand area of each biomass size ( $m$ and $n$ ). Note, in (d) Delta RS_ratio $=0$ when delta_size $=0$, but varies largely in a small region around 0. See Equation SI 4 for details.

\section{Root biomass prediction with age as a predictor}

When age is fixed as a predictor in the random forest model, the "best" trained model incorporates 14 additional predictors which are shoot biomass, height, soil nitrogen, $\mathrm{pH}$, bulk density, clay content, sand content, base saturation, cation exchange capacity, vapor pressure, mean annual precipitation, mean annual temperature, aridity and water table depth. This model slightly reduced the mean absolute error ( $\mathrm{MAE}=2.16$ vs. 2.18 ). Global total root biomass from this model is similar to the model without age. The age map is merged from several different sources (see Method), which likely introduce additional uncertainty in our estimation. We 


\section{Reference}

1 Olson, D. M. \& Dinerstein, E. The Global 200: Priority ecoregions for global conservation. (PDF file) Annals of the Missouri Botanical Garden 89:125-126. -The Nature Conservancy, USDA Forest Service and U.S. Geological Survey, based on Bailey, Robert G. 1995. Description of the ecoregions of the United States (2nd ed.). Misc. Pub. No. 1391, Map scale 1:7,500,000. USDA Forest Service. 108pp. -The Nature Conservancy (2003), based on Wiken, E.B. (compiler). 1986. Terrestrial ecozones of Canada. Ecological Land Classification Series No. 19. Environment Canada, Hull, Que. 26 pp. + map. (2002).

2 Hu, T. Y. et al. Mapping Global Forest Aboveground Biomass with Spaceborne LiDAR, Optical Imagery, and Forest Inventory Data. Remote Sensing 8, doi:10.3390/rs8070565 (2016).

3 Liu, Y. Y. et al. Recent reversal in loss of global terrestrial biomass. Nature Climate Change 5, 470-474, doi:10.1038/nclimate2581 (2015).

4 Hengl, T. et al. SoilGrids250m: Global gridded soil information based on machine learning. Plos One 12, doi:10.1371/journal.pone.0169748 (2017).

5 Batjes, N. H. (ISRIC - World Soil Information), WISE derived soil properties on a 30 by 30 arc-seconds global grid. https://data.isric.org/geonetwork/srv/api/records/dc7b283a-8f19-45e1-aaede9bd515119bc. (2015).

6 Hansen, M. C. et al. High-Resolution Global Maps of 21st-Century Forest Cover Change. Science 342, 850-853, doi:10.1126/science.1244693 (2013).

7 Jackson, R. B., Mooney, H. A. \& Schulze, E. D. A global budget for fine root biomass, surface area, and nutrient contents. Proceedings of the National Academy of Sciences of the United States of America 94, 7362-7366, doi:10.1073/pnas.94.14.7362 (1997).

8 Mokany, K., Raison, R. J. \& Prokushkin, A. S. Critical analysis of root: shoot ratios in terrestrial biomes. Global Change Biology 12, 84-96, doi:10.1111/j.13652486.2005.001043.x (2006).

9 Robinson, D. Scaling the depths: below-ground allocation in plants, forests and biomes. Functional Ecology 18, 290-295, doi:10.1111/j.0269-8463.2004.00849.x (2004).

10 Robinson, D. Implications of a large global root biomass for carbon sink estimates and for soil carbon dynamics. Proceedings of the Royal Society B-Biological Sciences 274, 2753-2759, doi:10.1098/rspb.2007.1012 (2007).

11 Saatchi, S. S. et al. Benchmark map of forest carbon stocks in tropical regions across three continents. Proceedings of the National Academy of Sciences of the United States of America 108, 9899-9904, doi:10.1073/pnas.1019576108 (2011).

12 Thurner, M. et al. Carbon stock and density of northern boreal and temperate forests. Global Ecology and Biogeography 23, 297-310, doi:10.1111/geb.12125 (2014).

13 Santoro, M. e. a. GlobBiomass - global datasets of forest biomass. PANGAEA, https://doi.org/10.1594/PANGAEA.894711. (2018).

14 Crowther, T. W. et al. Mapping tree density at a global scale. Nature 525, 201-+, doi:10.1038/nature14967 (2015). 
15 Saugier, B., Roy, J. \& Mooney, H. A. Estimations of global terrestrial productivity: converging toward a single number? In: Terrestrial Global Productivity (eds Roy J, Saugier B, Mooney HA), pp. 543-556. Academic Press, San Diego. (2001). 333, 988-993, doi:10.1126/science.1201609 (2011).

Pan, Y. D., Birdsey, R. A., Phillips, O. L. \& Jackson, R. B. in Annual Review of Ecology, Evolution, and Systematics, Vol 44 Vol. 44 Annual Review of Ecology Evolution and Systematics (ed D. J. Futuyma) 593-+ (2013).

18 Baccini, A. et al. Tropical forests are a net carbon source based on aboveground measurements of gain and loss. Science 358, 230-233, doi:10.1126/science.aam5962 (2017).

19 Jiang, Y. T. \& Wang, L. M. Pattern and control of biomass allocation across global forest ecosystems. Ecology and Evolution 7, 5493-5501, doi:10.1002/ece3.3089 (2017).

20 Niklas, K. J. Modelling below- and above-ground biomass for non-woody and woody plants. Annals of Botany 95, 315-321, doi:10.1093/aob/mci028 (2005).

21 Cairns, M. A., Brown, S., Helmer, E. H. \& Baumgardner, G. A. Root biomass allocation in the world's upland forests. Oecologia 111, 1-11, doi:10.1007/s004420050201 (1997).

22 Niklas, K. J. \& Enquist, B. J. On the vegetative biomass partitioning of seed plant leaves, stems, and roots. American Naturalist 159, 482-497, doi:10.1086/339459 (2002).

23 Liu, Y., Liu, R. G. \& Chen, J. M. Retrospective retrieval of long-term consistent global leaf area index (1981-2011) from combined AVHRR and MODIS data. Journal of Geophysical Research-Biogeosciences 117, doi:10.1029/2012jg002084 (2012).

24 Zhu, Z. C. et al. Global Data Sets of Vegetation Leaf Area Index (LAI)3g and Fraction of Photosynthetically Active Radiation (FPAR)3g Derived from Global Inventory Modeling and Mapping Studies (GIMMS) Normalized Difference Vegetation Index (NDVI3g) for the Period 1981 to 2011. Remote Sensing 5, 927-948, doi:10.3390/rs5020927 (2013).

25 Butler, E. E. et al. Mapping local and global variability in plant trait distributions. Proceedings of the National Academy of Sciences of the United States of America 114, E10937-E10946, doi:10.1073/pnas.1708984114 (2017).

26 Abramoff, R. Z. \& Finzi, A. C. Are above- and below-ground phenology in sync? New Phytologist 205, 1054-1061, doi:10.1111/nph.13111 (2015).

27 West, G. B., Brown, J. H. \& Enquist, B. J. A general model for the origin of allometric scaling laws in biology. Science 276, 122-126, doi:10.1126/science.276.5309.122 (1997).

28 West, G. B., Brown, J. H. \& Enquist, B. J. A general model for the structure and allometry of plant vascular systems. Nature 400, 664-667 (1999).

29 Farrior, C. E., Bohlman, S. A., Hubbell, S. \& Pacala, S. W. Dominance of the suppressed: Power-law size structure in tropical forests. Science 351, 155-157, doi:10.1126/science.aad0592 (2016).

30 Niklas, K. J. A phyletic perspective on the allometry of plant biomass-partitioning patterns and functionally equivalent organ-categories. New Phytologist 171, 27-40, doi:10.1111/j.1469-8137.2006.01760 (2006). 
474

475

476 